\title{
Systematic Analysis in Caenorhabditis elegans Reveals that the Spindle Checkpoint Is Composed of Two Largely Independent Branches
}

\author{
Anthony Essex, ${ }^{*+}$ Alexander Dammermann, ${ }^{*}$ Lindsay Lewellyn, ${ }^{*+}$ \\ Karen Oegema, ${ }^{*+}$ and Arshad Desai ${ }^{*+}$
}

\author{
*Department of Cellular and Molecular Medicine, Ludwig Institute for Cancer Research, University of \\ California, San Diego, La Jolla, CA 92093; and 'Biomedical Sciences Graduate Program, School of Medicine, \\ University of California, San Diego, La Jolla, CA 92093
}

Submitted October 21, 2008; Revised December 3, 2008; Accepted December 5, 2008

Monitoring Editor: Kerry S. Bloom

\begin{abstract}
Kinetochores use the spindle checkpoint to delay anaphase onset until all chromosomes have formed bipolar attachments to spindle microtubules. Here, we use controlled monopolar spindle formation to systematically define the requirements for spindle checkpoint signaling in the Caenorhabditis elegans embryo. The results, when interpreted in light of kinetochore assembly epistasis analysis, indicate that checkpoint activation is coordinately directed by the NDC-80 complex, the Rod/Zwilch/Zw10 complex, and BUB-1-three components independently targeted to the outer kinetochore

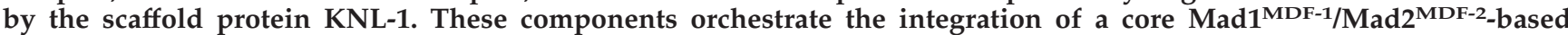

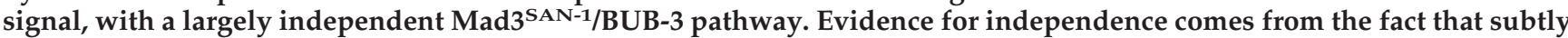
elevating Mad2 ${ }^{\mathrm{MDF}-2}$ levels bypasses the requirement for BUB-3 and Mad3 ${ }^{\mathrm{AN}-1}$ in kinetochore-dependent checkpoint activation. Mad3 $3^{\mathrm{SAN}-1}$ does not accumulate at unattached kinetochores and BUB-3 kinetochore localization is independent of Mad2 ${ }^{\mathrm{MDF}-2}$. We discuss the rationale for a bipartite checkpoint mechanism in which a core Mad1 ${ }^{\mathrm{MDF}-1} / \mathrm{Mad2}^{\mathrm{MDF}-2}$

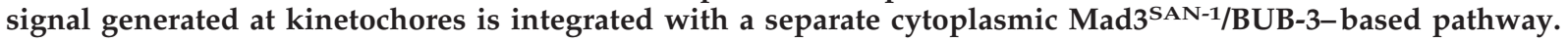

\section{INTRODUCTION}

Kinetochores assemble on centromeric DNA to connect spindle microtubules to sister chromatids and enable their segregation (Cheeseman and Desai, 2008). Improper segregation can generate aneuploid daughter cells, which in turn may promote apoptosis or tumorigenesis (Rajagopalan and Lengauer, 2004). To prevent aneuploidy, a kinetochorebased signaling pathway called the spindle checkpoint monitors chromosome-microtubule attachments and inhibits anaphase onset until all chromosomes have successfully bioriented, i.e., the two sister chromatids have attached to spindle microtubules emanating from opposing spindle poles (Musacchio and Salmon, 2007). The presence of even a single unattached kinetochore is sufficient to inhibit progression into anaphase in somatic cells (Rieder et al., 1995).

Screens for budding yeast mutants unable to arrest in the presence of microtubule-depolymerizing drugs identified as mitotic arrest deficient (Mad)1, Mad2, and Mad3 and budding $u$ ninhibited by benzimidazole (Bub)1 and Bub3 as molecular components of the checkpoint (Hoyt et al., 1991; Li and Murray, 1991). Mps1, a kinase essential for spindle pole body duplication, was subsequently also shown to be required for the checkpoint (Weiss and Winey, 1996). Verte-

This article was published online ahead of print in $M B C$ in Press (http://www.molbiolcell.org/cgi/doi/10.1091/mbc.E08-10-1047) on December 24, 2008.

Address correspondence to: Arshad Desai (abdesai@ucsd.edu).

Abbreviations used: DCON, decondensation; NEBD, nuclear envelope breakdown; OCC, onset of cortical contractility. brates and flies have additional proteins essential for checkpoint signaling, including Rod, Zwilch, and Zw10 (RZZ), which copurify as a complex and are interdependent for their kinetochore localization (Williams et al., 2003; Buffin et al., 2005; Karess, 2005; Kops et al., 2005), and the kinesin-like motor protein CENP-E (Abrieu et al., 2001). Another difference between vertebrates and yeast is that the Mad3-like vertebrate protein BubR1 contains a C-terminal Bub1-like kinase domain (Murray and Marks, 2001). Localization interdependencies, turnover dynamics, and biochemical interactions among the checkpoint proteins have been primarily studied in vertebrates and yeast and indicate that Bub1 is at the top of the checkpoint protein kinetochore localization hierarchy (Sharp-Baker and Chen, 2001; Gillett et al., 2004; Johnson et al., 2004; Meraldi et al., 2004; Rischitor et al., 2007) and that downstream components such as Mad2 are rapidly exchanging at unattached kinetochores to communicate the checkpoint signal to the cytoplasm (Musacchio and Salmon, 2007).

Checkpoint activation delays sister chromatid separation and mitotic exit by preventing the anaphase-promoting complex/cyclosome (APC/C), an E3-ubiquitin ligase, from inducing the destruction of securin and cyclin B (Peters, 2002; Yu, 2002). The checkpoint sequesters or inhibits Cdc20 (Hwang et al., 1998; Kim et al., 1998), which is essential for APC/C activation and substrate recognition (Yu, 2007). The precise mechanism of Cdc20 inhibition by the checkpoint is a current topic of investigation. Recent structural and in vitro studies have shown that a kinetochore-bound Mad1Mad2 complex interacts with free Mad2 and modifies its conformation to make it a more potent inhibitor of APCCdc20 (Sironi et al., 2002; Luo et al., 2004; De Antoni et al., 
Table 1. Worm strains used in this study

\begin{tabular}{|c|c|c|}
\hline Strain no. & Genotype & Label in figures and movies \\
\hline TH32 & unc-119(ed3) III; ruIs32 [pAZ132; pie-1/GFP::histone H2B] III; ddIs6 [GFP::tbg-1; unc-119(+)] & $\begin{array}{l}\text { GFP-histone } \mathrm{H} 2 \mathrm{~b} ; \mathrm{GFP}-\gamma- \\
\text { tubulin }\end{array}$ \\
\hline OD56 & unc-119(ed3) III; ltIs37 [pAA64; pie-1/mCherry::his-58; unc-119 (+)] IV & mCherry-histone $\mathrm{H} 2 \mathrm{~b}$ \\
\hline OD95 & $\begin{array}{l}\text { unc-119(ed3) III; ltIs37 [pAA64; pie-1/mCherry::his-58; unc-119 (+)] IV; ltIs38 [pAA1; } \\
\text { pie-1/GFP::PH(PLC1delta1); unc-119(+)] }\end{array}$ & mCherry-histone H2b; GFP-PH \\
\hline OD108 & unc-119(ed3) III; ltIs52 [pOD379; pie-1/GFP::Y69A2AR.30; unc-119 (+)] & GFP-Mad2 ${ }^{\mathrm{MDF}-2}$ \\
\hline OD109 & unc-119(ed3) III; ltIs53 [pOD380; pie-1/GFP::ZC328.4; unc-119 (+)] & GFP-Mad3 $3^{\text {SAN-1 }}$ \\
\hline OD110 & $\begin{array}{l}\text { unc-119(ed3) III; ltIs52 [pOD379; pie-1/GFP::Y69A2AR.30; unc-119 (+)]; ltIs37 [pAA64; } \\
\text { pie-1/mCherry::his-58; unc-119 (+)] IV }\end{array}$ & $\begin{array}{l}\text { mCherry-histone H2b; GFP- } \\
\text { Mad2 }\end{array}$ \\
\hline OD133 & unc-119(ed3) III; ltIs73 [pOD377; pie-1/GFP::Y54G9A.6; unc-119 (+)] & GFP-BUB-3 \\
\hline OD196 & $\begin{array}{l}\text { unc-119(ed3) III; ltIs73 [pOD377; pie-1/GFP::Y54G9A.6; unc-119 (+)], ltIs37 [pAA64; } \\
\text { pie-1/mCherry::his-58; unc-119 (+)] IV }\end{array}$ & $\begin{array}{l}\text { mCherry-histone H2b; GFP- } \\
\text { BUB-3 }\end{array}$ \\
\hline OD197 & $\begin{array}{l}\text { unc-119(ed3) III; ltIs53 [pOD380; pie-1/GFP::ZC328.4; unc-119 (+)], ltIs37 [pAA64; } \\
\quad \text { pie-1/mCherry::his-58; unc-119 (+)] IV }\end{array}$ & $\begin{array}{l}\text { mCherry-histone H2b; GFP- } \\
\text { Mad3 }^{\text {SAN-1 }}\end{array}$ \\
\hline OD206 & unc-119(ed3) III; ltIs37 [pAA64; pie-1/mCHERRY::his-58; unc-119 (+)] IV; san-1(ok1580) I & 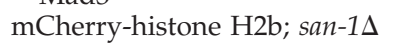 \\
\hline OD207 & $\begin{array}{l}\text { unc-119(ed3) III; ltIs52 [pOD379; pie-1/GFP::Y69A2AR.30; unc-119 (+)], ltIs37 [pAA64; } \\
\text { pie-1/mCherry::his-58; unc-119 (+)] IV, san-1(ok1580) I }\end{array}$ & 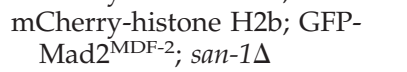 \\
\hline OD208 & $\begin{array}{l}\text { unc-119(ed3) III; ltIs53 [pOD380; pie-1/GFP::ZC328.4; unc-119 (+)], ltIs37 [pAA64; } \\
\text { pie-1/mCherry::his-58; unc-119 (+)] IV, san-1(ok1580) I }\end{array}$ & 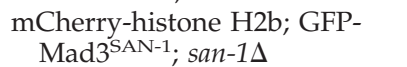 \\
\hline OD215 & unc-119(ed3) III; ltIs37 [pAA64; pie-1/mCHERRY::his-58; unc-119 (+)] IV; mdf-2(tm2190) & mCherry-histone $\mathrm{H} 2 \mathrm{~b} ; m d f-2 \Delta$ \\
\hline OD216 & $\begin{array}{l}\text { unc-119(ed3) III; ltIs52 [pOD379; pie-1/GFP::Y69A2AR.30; unc-119 (+)], ltIs37 [pAA64; } \\
\text { pie-1/mCherry::his-58; unc-119 (+)] IV, mdf-2(tm2190) }\end{array}$ & $\begin{array}{l}\text { mCherry-histone H2b; GFP- } \\
\text { Mad2 }{ }^{\mathrm{MDF}-2} ; m d f-2 \Delta\end{array}$ \\
\hline RB1391 & san-1(ok1580) I & N/A-Source strain for san-1s \\
\hline AG170 & $m d f-2(t m 2190)$ & N/A-Source strain for $m d f-2 \Delta$ \\
\hline
\end{tabular}

2005; Vink et al., 2006; Mapelli et al., 2007; Yang et al., 2008). However, Mad2 is unlikely to be the sole Cdc20 inhibitor. BubR1 has been shown to directly bind Cdc20 and subunits of the APC/C (Tang et al., 2001; Sironi et al., 2002). Bub1 has also been shown to bind and phosphorylate Cdc20 (Tang et al., 2004a). Finally, a complex named mitotic checkpoint complex containing BubR1 (Mad3 in yeast and worms), Bub3, Mad2 and Cdc20 that displays much higher APC/C inhibitory activity than purified $\mathrm{Mad} 2$ in vitro has been purified from HeLa cells as well as budding yeast (Hardwick et al., 2000; Fraschini et al., 2001; Sudakin et al., 2001).

The early C. elegans embryo has emerged as an important model for studying kinetochore assembly and function. In vivo assembly epistasis analysis has comprehensively defined the relationships between kinetochore constituents, including proteins that direct assembly of centromeric chromatin (Maddox et al., 2007) and proteins that provide the core microtubule binding activity of the kinetochore (Desai et al., 2003; Cheeseman et al., 2004, 2006). These studies revealed a central role for the scaffold-like protein KNL-1 in outer kinetochore assembly, including the targeting of Bub1, the upstream kinase involved in spindle checkpoint activation (Desai et al., 2003). The role of KNL-1 family proteins in checkpoint signaling is conserved in vertebrates (Kittler et al., 2007; Kiyomitsu et al., 2007). A delay in mitosis after treatment with microtubule-depolymerizing drugs has been documented in the gonad and in embryos (Kitagawa and Rose, 1999; Nystul et al., 2003; Encalada et al., 2005; Stein et al., 2007; Tarailo et al., 2007; Hajeri et al., 2008), and spindle checkpoint proteins have been implicated in cessation of activity under anoxia (Nystul et al., 2003) and starvationinduced arrest of germ cell precursors (Watanabe et al., 2008).

Here, we develop a controlled monopolar spindle formation-based assay in the early C. elegans embryo to systematically analyze the relationship between kinetochore structure and checkpoint activation. Our results indicate that checkpoint activation is coordinately directed by three com- ponents-the NDC-80 complex, the Rod/Zwilch/Zw10 complex, and BUB-1-that are targeted independently of one another by the outer kinetochore scaffold protein KNL-1. Mad3 $3^{\text {SAN-1, }}$, unlike the other checkpoint proteins, does not enrich at unattached kinetochores. Surprisingly, a subtle (2.5-fold) increase in Mad2 ${ }^{\mathrm{MDF}-2}$ levels can bypass the requirement of Mad3 $3^{\mathrm{SAN}-1}$ as well as BUB-3 for checkpoint activation. We propose that a core Mad1 ${ }^{\mathrm{MDF}-1} / \mathrm{Mad}^{\mathrm{MDF}-2}$ signal generated at kinetochores is integrated with a largely independent cytoplasmic Mad3 $3^{\text {SAN-1 }}$ /BUB-3-based signal to achieve APC/C inhibition.

\section{MATERIALS AND METHODS}

\section{Strains and Culture Conditions}

All C. elegans strains were maintained at $20^{\circ} \mathrm{C}$. Strain genotypes are listed in Table 1. The strains OD108 (expressing a GFP fusion with MDF-2), OD109 (expressing a GFP fusion with SAN-1), and OD133 (expressing a GFP fusion with BUB-3) were all generated by cloning the coding (BUB-3 and MDF-2) or genomic (SAN-1) sequences into the Spe1 site of pIC26 (Cheeseman et al., 2004) and by integrating the constructs into DP38 [unc-119 (ed3)] by ballistic bombardment (Praitis et al., 2001) with a PDS-1000/He Biolistic Particle Delivery System (Bio-Rad, Hercules, CA). Fluorescence intensity measurements in the nuclear region during early prometaphase (immediately after nuclear envelope breakdown [NEBD]) in the AB cell indicate that the GFP::Mad3 $3^{\mathrm{SAN}-1}$ and GFP::BUB-3 proteins are expressed at similar levels (mean \pm SD in arbitrary units: $100 \pm 16[\mathrm{n}=8]$ for GFP::Mad3 ${ }^{\text {SAN-1 }}$ and $81 \pm$ $22\left[\mathrm{n}=9\right.$ ] for GFP-BUB-3) and that the GFP::Mad2 ${ }^{\mathrm{MDF}-2}$ protein is expressed at an approximately threefold higher level relative to the other two $(300 \pm 44$ $[\mathrm{n}=16])$. The strain RB1391 [san-1(ok1580) I; referred to as Mad3 ${ }^{\operatorname{san}^{-1} \Delta} \Delta$ ] was obtained from the CGC. The strain AG170 was a generous gift from the laboratory of Dr. A. Golden. Two-color strains were constructed by mating as described previously (Green et al., 2008).

\section{RNA Interference (RNAi)}

Double-stranded RNA (dsRNA) was prepared as described previously (Oegema et al., 2001). Oligonucleotides used for dsRNA production are listed in Table 2. L4 worms were injected with dsRNA and incubated for $45-48 \mathrm{~h}$ at $20^{\circ} \mathrm{C}$. For double depletions, dsRNAs were mixed to obtain equal concentrations of $>0.75 \mathrm{mg} / \mathrm{ml}$ for each RNA. Western blots were performed as described previously (Desai et al., 2003). 
Table 2. Double-stranded RNAs used in this study

\begin{tabular}{|c|c|c|c|c|c|c|}
\hline RNA no. & Gene no. & Name & $\begin{array}{l}\text { Conc. } \\
\mathrm{mg} / \mathrm{ml}\end{array}$ & Oligo \#1 & Oligo \#2 & Template \\
\hline 2 & Y69A2AR.30 & $m d f-2$ & 3.2 & $\begin{array}{l}\text { TAATACGACTCACTAT } \\
\text { AGGgagaccacacggat } \\
\text { gtaaagacacaaaacg }\end{array}$ & $\begin{array}{l}\text { TAATACGACTCACTATA } \\
\text { GGgagaccacgtgaact } \\
\text { gacgtcgagaatgag }\end{array}$ & cDNA \\
\hline 3 & F55G1.4 & rod -1 & 2.1 & $\begin{array}{l}\text { TAATACGACTCACTATAGG } \\
\text { gagaccactcgtatggaaagtatgccactg }\end{array}$ & $\begin{array}{l}\text { TAATACGACTCACTATAGG } \\
\text { gagaccacgttcatgcaaagcagtcaaatc }\end{array}$ & cDNA \\
\hline 5 & F20D12.4 & $c z w-1$ & 2.0 & $\begin{array}{l}\text { TAATACGACTCACTATAGG } \\
\text { gagaccactgattggacaattaccagaacg }\end{array}$ & $\begin{array}{l}\text { TAATACGACTCACTATAGG } \\
\text { gagaccacctgattgtcaccactagcctca }\end{array}$ & cDNA \\
\hline 29 & T06E4.1 & $h c p-2$ & 1.9 & $\begin{array}{l}\text { TAATACGACTCACTATAGG } \\
\text { tctcggaaaggaatcgaaaa }\end{array}$ & $\begin{array}{l}\text { AATTAACCCTCACTAAAGG } \\
\text { tcgttgtctccaattccaca }\end{array}$ & genomic DNA \\
\hline 61 & C02F5.1 & $k n l-1$ & 1.9 & $\begin{array}{l}\text { TAATACGACTCACTATAGG } \\
\text { ccgctgaaatggatacgagt }\end{array}$ & $\begin{array}{l}\text { AATTAACCCTCACTAAAGG } \\
\text { ccatgctaatgtcttcacacg }\end{array}$ & genomic DNA \\
\hline 62 & K11D9.1 & $k l p-7$ & 2.6 & $\begin{array}{l}\text { TAATACGACTCACTATAGG } \\
\text { gtgcttctgccaacaaacg }\end{array}$ & $\begin{array}{l}\text { AATTAACCCTCACTAAAGGt } \\
\text { gatctggaatatggcgtga }\end{array}$ & genomic DNA \\
\hline 64 & ZK1055.1 & $h c p-1$ & 2.1 & $\begin{array}{l}\text { TAATACGACTCACTATAGG } \\
\text { aaaccgagtcgccattttc }\end{array}$ & $\begin{array}{l}\text { AATTAACCCTCACTAAAGG } \\
\text { agatcgcgctgaagactttc }\end{array}$ & genomic DNA \\
\hline 84 & W01B6.9 & $n d c-80$ & 1.6 & $\begin{array}{l}\text { AATTAACCCTCACTAAAGG } \\
\text { ccccagtctgagtcaacctc }\end{array}$ & $\begin{array}{l}\text { TAATACGACTCACTATAGG } \\
\text { ccaactcgctttgaatttcc }\end{array}$ & genomic DNA \\
\hline 93 & T03F1.9 & $h c p-4$ & 1.36 & $\begin{array}{l}\text { AATTAACCCTCACTAAAGG } \\
\text { ggaaatgtacggagcgaaaa }\end{array}$ & $\begin{array}{l}\text { TAATACGACTCACTATAG } \\
\text { gacattgttggtgggtccaat }\end{array}$ & genomic DNA \\
\hline 205 & F59E12.2 & $z y g-1$ & 1.4 & $\begin{array}{l}\text { AATTAACCCTCACTAAAGG } \\
\text { tggacggaaattcaaacgat }\end{array}$ & $\begin{array}{l}\text { TAATACGACTCACTATAGG } \\
\text { aacgaaattccettgagctg }\end{array}$ & cDNA \\
\hline 235 & Y39G10AR.2 & $z w l-1$ & 2.0 & $\begin{array}{l}\text { AATTAACCCTCACTAAAGG } \\
\text { atgccactcaccatcgagcag }\end{array}$ & $\begin{array}{l}\text { TAATACGACTCACTATAGG } \\
\text { ggatcagtgaagcgagatgactc }\end{array}$ & cDNA \\
\hline 263 & C50F4.11 & $m d f-1$ & 1.12 & $\begin{array}{l}\text { TAATACGACTCACTATAGG } \\
\text { aagcgaagttggctgaaaaa }\end{array}$ & $\begin{array}{l}\text { AATTAACCCTCACTAAAGGag } \\
\text { catcctcaagtcgttcgt }\end{array}$ & genomic DNA \\
\hline 264 & Y54G9A.6 & $b u b-3$ & .875 & $\begin{array}{l}\text { TAATACGACTCACTATAGG } \\
\text { gacgctaaaacttgtcggat }\end{array}$ & $\begin{array}{l}\text { AATTAACCCTCACTAAAGG } \\
\text { ttatgaagctgaataatacg }\end{array}$ & genomic DNA \\
\hline 265 & ZC328.4 & san-1 & 1.23 & $\begin{array}{l}\text { TAATACGACTCACTATAGG } \\
\text { cgaagaacttcaaaacctgga }\end{array}$ & $\begin{array}{l}\text { AATTAACCCTCACTAAAGG } \\
\text { tttgtcggtccagatcettc }\end{array}$ & genomic DNA \\
\hline 327 & Y45F10D.9 & sas- 6 & 3.7 & $\begin{array}{l}\text { AATTAACCCTCACTAAAGG } \\
\text { tatggagctaatttgaactcggtta }\end{array}$ & $\begin{array}{l}\text { TAATACGACTCACTATAGG } \\
\text { agcagagttttattttcaagtaaagga }\end{array}$ & genomic DNA \\
\hline 358 & F58A4.3 & $h c p-3$ & 2.85 & $\begin{array}{l}\text { AATTAACCCTCACTAAAGG } \\
\text { gccgatgacacccaattat }\end{array}$ & $\begin{array}{l}\text { TAATACGACTCACTATAGG } \\
\text { ccgtgggagtaatcgacaag }\end{array}$ & cDNA \\
\hline 365 & R06C7.8 & $b u b-1$ & 3.4 & $\begin{array}{l}\text { AATTAACCCTCACTAAAGG } \\
\text { tgccaaatggaaggacactt }\end{array}$ & $\begin{array}{l}\text { TAATACGACTCACTATAGG } \\
\text { tctgagattcttccggttcg }\end{array}$ & genomic DNA \\
\hline 374 & & GFP & 1.1 & $\begin{array}{l}\text { TAATACGACTCACTATAGG } \\
\text { gtcagtggagaggg tggaaggtg }\end{array}$ & $\begin{array}{l}\text { AATTAACCCTCACTAAAGG } \\
\text { catgccatgtgtaatcccagcagc }\end{array}$ & Plasmid pIC26 \\
\hline
\end{tabular}

\section{Microscopy}

All images for the timing assays and immunofluorescence were acquired on a DeltaVision deconvolution microscope (Applied Precision, Issaquah, WA) equipped with a CoolSNAP charge-coupled device camera (Roper Scientific, Trenton, NJ) at $20^{\circ} \mathrm{C}$. Z-sections were acquired at $2-\mu \mathrm{m}$ steps by using a $100 \times$, 1.3 numerical aperture (NA) Olympus U-Planapo objective with $2 \times 2$ binning and a $480 \times 480$ pixel area at 20-s intervals, and each exposure was $100 \mathrm{~ms}$. Z-stacks were projected and imported into MetaMorph (Molecular Devices, Sunnyvale, CA) to rotate and scale images. Immunofluorescence was performed as described previously (Oegema et al., 2001; Desai et al., 2003). Polyclonal antibodies against BUB-1, BUB-3 (amino acids 189-329) Zwilch ZWL-1 (amino acids 1-200), and Mad2 ${ }^{\mathrm{MDF}-2}$ (splice variant Y39A2AR.30A amino acids 2-203) were generated as described previously (Oegema et al., 2001; Desai et al., 2003). All images acquired using a specific strain or specific antibody were scaled identically.

For GFP::BUB-3, GFP::Mad3 ${ }^{\mathrm{SAN}-1}$, and GFP::Mad2MDF-2 localization, embryos were filmed using a spinning disk confocal mounted on an inverted microscope (TE2000-E; Nikon, Tokyo, Japan) equipped with a $60 \times 1.4$ NA Plan Apochromat lens (Nikon), a krypton-argon 2.5-W water-cooled laser (Spectra Physics, San Jose, CA), and an electron multiplication back-thinned charge-coupled device camera (iXon; Andor Technology, Belfast, Ireland). Acquisition parameters, shutters, and focus were controlled by MetaMorph software (MDS Analytical Technologies, Winnersh, United Kingdom). Then, $5 \times 1 \mu \mathrm{m}$ RFP/GFP $\mathrm{z}$-series with no binning and a single central reference differential interference contrast (DIC) image with no binning were collected every $20 \mathrm{~s}$. Exposures were $300 \mathrm{~ms}$ for both green fluorescent protein (GFP) and red fluorescent protein (RFP), and $200 \mathrm{~ms}$ for DIC (laser power, 50\%).

To specifically measure kinetochore-localized GFP::Mad2 ${ }^{\mathrm{MDF}-2}$, a subtraction approach (Dammermann et al., 2008) was used. See Supplemental Figure 3 legend for details.

\section{RESULTS}

Controlled Monopolar Spindle Formation in the C. elegans Embryo Elicits a Cell Cycle Delay that Requires Conserved Spindle Checkpoint Components

To quantitatively monitor spindle checkpoint signaling in $C$. elegans embryos, we triggered checkpoint activation by generating monopolar spindles. In C. elegans, RNAi-mediated depletion of proteins required for centriole duplication results in a bipolar first division, which serves as a useful internal control, followed by subsequent monopolar divisions (Figure 1A; O'Connell et al., 2001). Monopolar spindles have both unattached kinetochores and kinetochores not under tension and have been shown to activate the checkpoint in other organisms (Kapoor et al., 2000). This approach avoids drug treatments, which are difficult due to the impermeable eggshell surrounding the embryos.

We quantified the time from NEBD to chromosome decondensation (DCON) in embryos expressing GFP-histone $\mathrm{H} 2 \mathrm{~b}$ (to mark the chromosomes) and GFP- $\gamma$-tubulin (to mark the spindle poles). NEBD was defined by diffusion of free GFP-histone $\mathrm{H} 2 \mathrm{~b}$ out of the nucleoplasm and DCON as the disappearance of fluorescent punctae throughout the decondensing chromatin (Figure 1B). Monopolar spindles were generated by depleting the kinase ZYG-1 or the cent- 
A
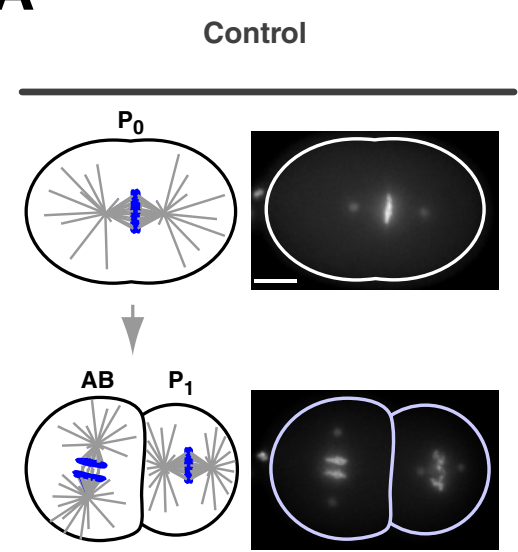

B
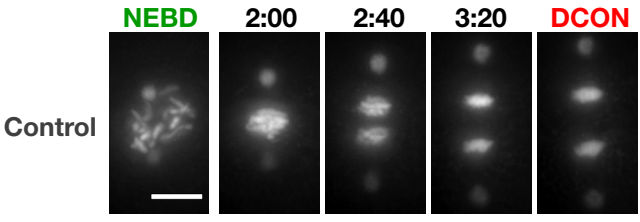

RNAi: NEBD

zyg-1
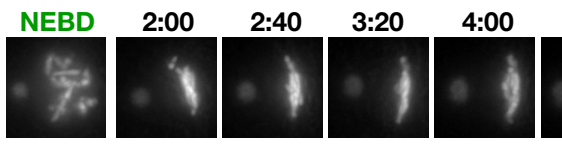

AB Cell
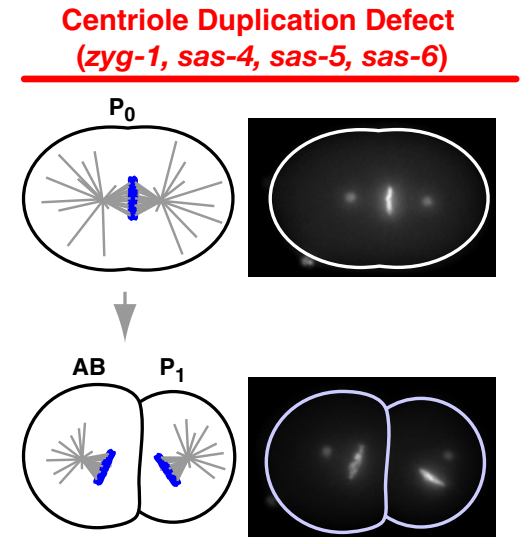

C
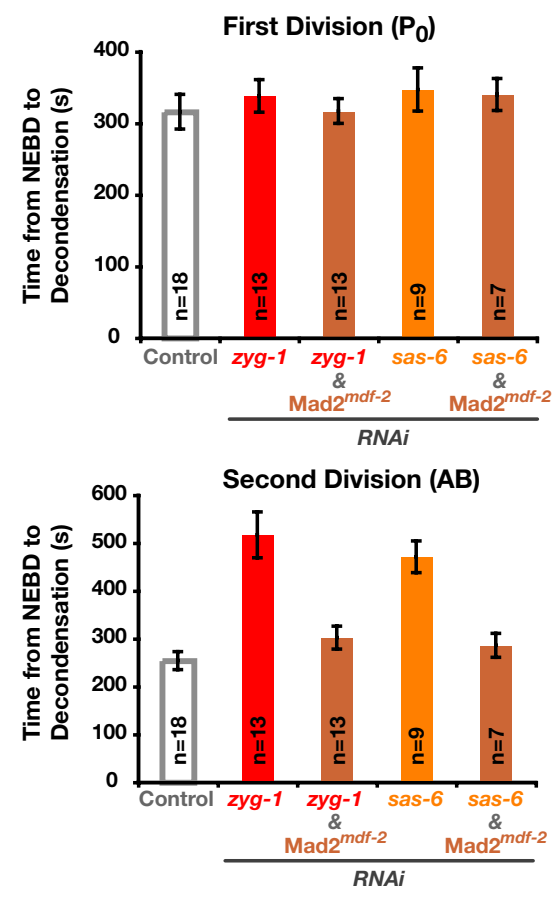

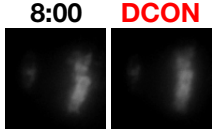

$\mathbf{E}$
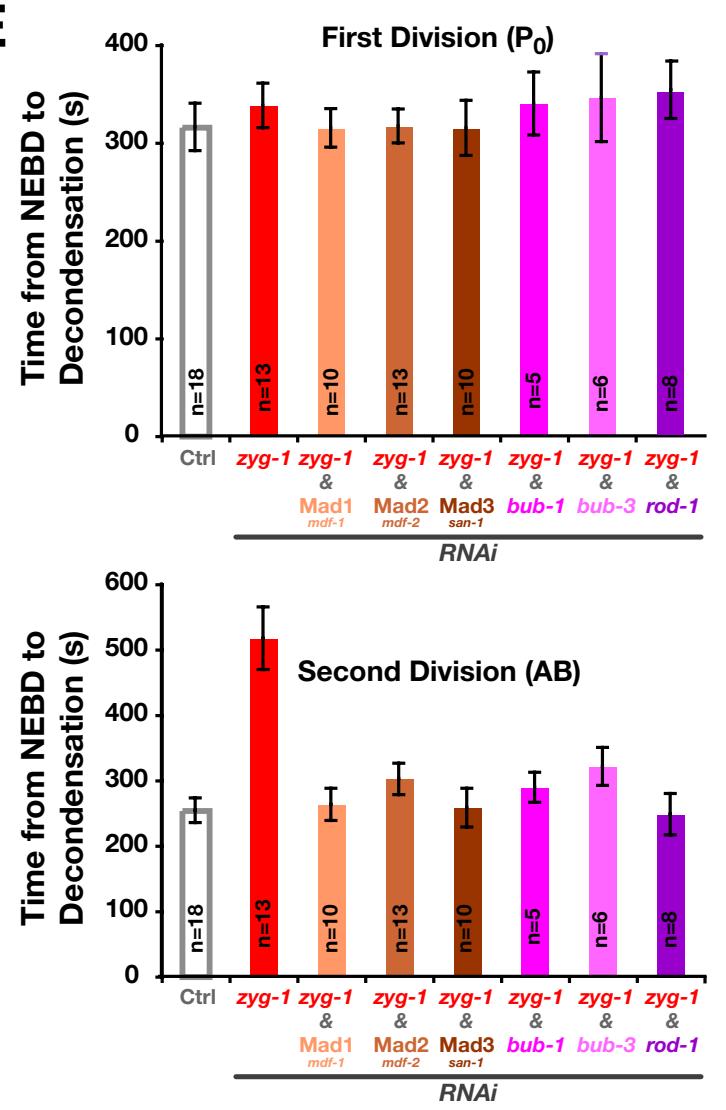

Figure 1. Controlled monopolar spindle formation in C. elegans embryos results in a spindle checkpoint-mediated cell cycle delay. (A) Selected frames from time-lapse sequences of the first $\left(\mathrm{P}_{0}\right)$, second $(\mathrm{AB})$, and third $\left(\mathrm{P}_{1}\right)$ divisions of embryos expressing GFP::histone $\mathrm{H} 2 \mathrm{~B}$ (arrow) and GFP:: $\gamma$-tubulin (arrowheads), accompanied by schematics to the left of each image. Control embryos (left) have bipolar mitotic 
riole structural protein SAS-6 (Bettencourt-Dias and Glover, 2007). In both control and centriole duplication-inhibited embryos, the timing of NEBD-DCON was unaltered in the first mitotic division. By contrast, the same interval in the subsequent monopolar mitotic divisions was significantly elongated in both the anterior $A B$ cell (Figure 1C) and the posterior P1 cell (data not shown). In all subsequent experiments, we only present analysis of mitotic timing in the first embryonic division and in the AB cell.

To determine whether the delay in cells with monopolar spindles was due to spindle checkpoint activation, we codepleted the conserved checkpoint protein Mad2 ${ }^{\mathrm{MDF}-2}$. Mad2 ${ }^{\mathrm{MDF}-2}$ codepletion did not affect the timing of the first bipolar division (Figure 1C and Supplemental Movie S1), but it abolished the cell cycle delay triggered by monopolar spindle formation (Figure 1, B and C, and Supplemental Movie S2). Mad2 ${ }^{\mathrm{MDF}-2}$ depletion on its own did not affect the NEBD-DCON interval in either division (Supplemental Figure S1A). Similar results were obtained for both ZYG-1- and SAS-6-depleted embryos, establishing that the delay in mitotic exit is due to the presence of monopolar spindles and not due to a specific role for the targeted proteins in cell cycle progression. We conclude that controlled generation of monopolar spindles elicits a Mad2 ${ }^{\mathrm{MDF}-2}$-dependent cell cycle progression delay in the C. elegans embryo.

The C. elegans homologues of proteins implicated in checkpoint signaling are indicated in Figure 1D together with the consequences of their RNAi-mediated depletion. C. elegans has a Mad3-like protein (Mad3 ${ }^{\mathrm{SAN}-1}$ ) instead of a BubR1-like kinase and lacks an Mps1-like kinase, which is also absent in other related nematodes with sequenced genomes. Unlike depletion of other checkpoint proteins, depletion of BUB-1, ROD-1, or Zwilch ${ }^{Z W L-1}$ resulted in penetrant embryonic lethality, reflecting functions for these proteins in chromosome segregation in addition to their role in checkpoint signaling. Depletion of $\mathrm{Zw} 10^{\mathrm{CZW}-1}$ resulted in penetrant sterility consistent with a previously described nonmitotic function for Zw10 (independently of Rod and Zwilch) in membrane trafficking (Hirose et al., 2004), which is required for oocyte production.

We next examined the consequences of depleting components of the spindle checkpoint pathway in the monopolar spindle assay. Individual depletions of each protein abolished the monopolar spindle-induced mitotic delay (Figure $1 \mathrm{E})$. By contrast, none of the depletions affected the timing of the first bipolar division (Figure 1E). Abolishing checkpoint signaling by depletion of Mad1 $1^{\mathrm{MDF}-1}$ also did not alter kin-

Figure 1 (cont). spindles in all divisions. In embryos depleted of proteins necessary for centriole duplication (right), the $\mathrm{P}_{0}$ cell assembles a bipolar spindle (with 1 sperm-derived centriole at each spindle pole), but the subsequent $\mathrm{AB}$ and $\mathrm{P}_{1}$ cells inherit only one centriole and assemble monopolar spindles. Bar, $10 \mu \mathrm{m}$. (B) Selected frames from time-lapse sequences of control, $z y g-1(R N A i)$, and $z y g$ $1+\mathrm{Mad}^{\text {maff-2 }}$ (RNAi) embryos expressing GFP::histone H2B and GFP:: $\gamma$-tubulin. Only the AB cell spindle region is shown. Numbers above panels indicate time after NEBD in minutes:seconds; DCON indicates time of chromosome decondensation. Bar, $5 \mu \mathrm{m}$. (C) The mean NEBD to DCON interval for the indicated conditions is plotted for both the first $\left(\mathrm{P}_{0}\right.$; top) and second (AB; bottom) mitotic divisions. (D) Summary of the effect of depleting the C. elegans orthologues of spindle checkpoint proteins on embryo viability. Larval (L)4 hermaphrodites were injected with dsRNAs, and the consequences on embryo viability were assessed 36-48 h after injection. (E) The mean NEBD to DCON interval for the indicated conditions is plotted for both the first $\left(\mathrm{P}_{0} ;\right.$ top) and second $(\mathrm{AB}$ bottom) mitotic divisions. Error bars are the $95 \%$ confidence interval. etochore-spindle microtubule interactions, as assessed by quantitative analysis of spindle pole separation (Supplemental Figure S1B; Oegema et al., 2001). We conclude that controlled monopolar spindle formation generates a reproducible spindle checkpoint-mediated cell cycle delay in the early C. elegans embryo.

\section{Systematic Analysis Subdivides the Protein Constituents of the Kinetochore into Three Classes Based on Their Roles in Spindle Checkpoint Activation}

The protein components of the C. elegans kinetochore can be partitioned into different functional groups. A set of three proteins (CENP-A ${ }^{\mathrm{HCP}-3}, \mathrm{CENP}-\mathrm{C}^{\mathrm{HCP}-4}$, and KNL-2) form the centromeric chromatin foundation for kinetochore assembly (Buchwitz et al., 1999; Moore and Roth, 2001; Oegema et al., 2001; Maddox et al., 2007). The conserved KNL-1/Mis12 complex/Ndc80 complex (KMN) network assembles on this foundation to form the core microtubule binding site of the kinetochore (Desai et al., 2003; Cheeseman et al., 2004, 2006). KNL-1 serves as a scaffold that recruits not only the microtubule-binding NDC-80 complex but also other outer kinetochore proteins such as the RZZ complex, the kinase BUB-1, the CENP-F-like proteins HCP-1/2, and the microtubulebinding protein CLASPCLS-2 (Desai et al., 2003).

To investigate their role in spindle checkpoint activation, we systematically analyzed the consequences of depleting kinetochore components on the monopolar spindle-induced cell cycle delay. Because the chromosome missegregation associated with several of these depletions made chromosome decondensation difficult to score, we used an alternative method to time cell cycle progression by measuring the interval from NEBD to onset of cortical contractility (OCC) in a strain coexpressing mCherry-Histone $\mathrm{H} 2 \mathrm{~b}$ and a GFPtagged plasma membrane marker (Figure 2B and Supplemental Movies S3 and S4). Cortical contractility is tightly linked to mitotic exit and is a frequently used visual marker in live imaging studies (Canman et al., 2000; Kurz et al., 2002). We defined OCC as the transition of the membrane from a roughly circular conformation to a rectangular conformation (in embryos with bipolar spindles) or to the appearance of membrane "blebs" (in embryos with monopolar spindles; Figure 2B, arrowheads). Using this assay, we confirmed that monopolar spindles trigger a Mad2 ${ }^{\mathrm{MDF}-2}$-dependent increase in the NEBD-OCC interval relative to controls (Figure 2C and Supplemental Movie S4).

Next, we depleted each of the kinetochore components on their own and in conjunction with ZYG-1 and measured the NEBD-OCC intervals for the first two mitotic divisions. None of the tested proteins affected the NEBD to OCC interval during the first bipolar mitotic division (Figure $2 \mathrm{C}$ and Supplemental Movie S3). By contrast, analysis of cell cycle timing in the $\mathrm{AB}$ cell partitioned the targeted kinetochore components into three classes. The first class (I), which includes CENP-A ${ }^{\mathrm{HCP}-3}$, CENP-C ${ }^{\mathrm{HCP}-4}$, KNL-3 (data not shown), KNL-1, and NDC-80, is composed of proteins required for the monopolar spindle-induced delay; depletions of proteins in this class did not result in a significant cell cycle delay relative to controls (Figure 2C). The second class (II), which includes MCAK KLP-7 and the nonessential kinetochore protein KBP-5 (Figure 2C; data not shown), was dispensable for the monopolar spindle-induced delay. The third class (III) includes proteins whose depletion induces a cell cycle delay on their own, regardless of whether spindles were bipolar or monopolar; HCP-1/2, which are functionally analogous to CENP-F in vertebrates (Moore and Roth, 2001; Cheeseman et al., 2005; Encalada et al., 2005; Tarailo et al., 2007; Hajeri et al., 2008), fell into this class (Figure 2C). 


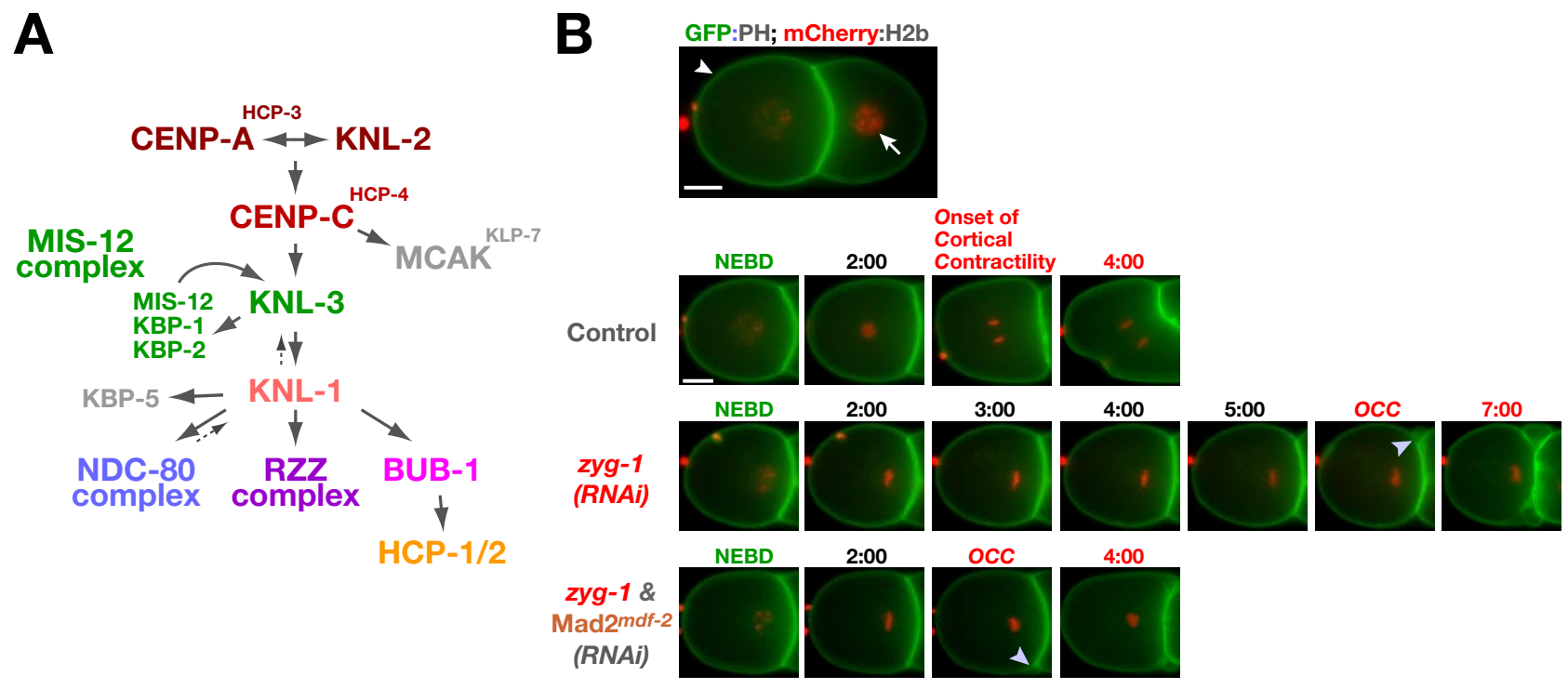

\section{C}
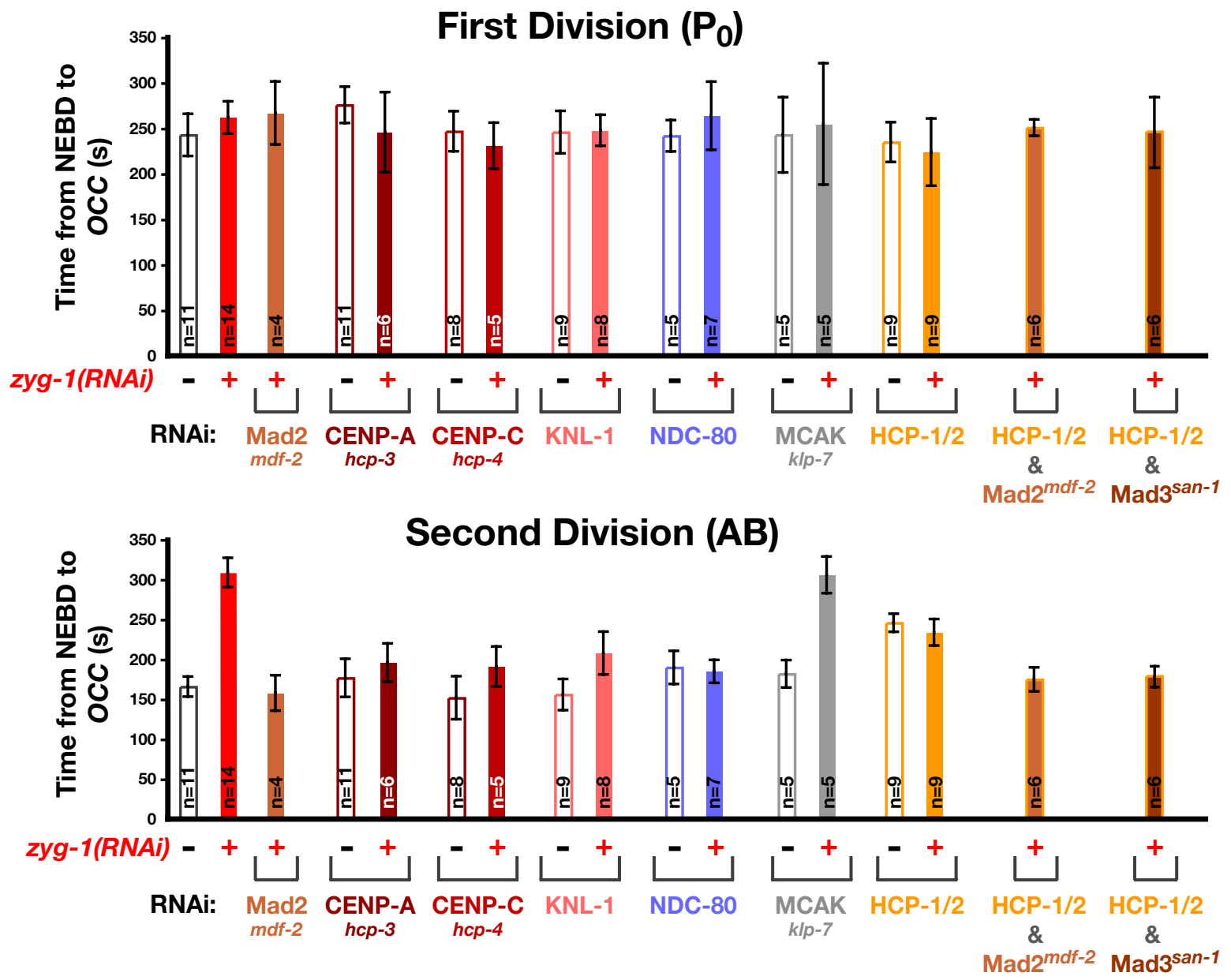

Figure 2. Systematic analysis comparing the position of components in the kinetochore assembly hierarchy to their role in checkpoint activation. (A) Summary of the kinetochore assembly pathway in C. elegans embryos. (B) Selected frames from time-lapse sequences of control, $z y g-1(R N A i)$, and $z y g-1+\mathrm{Mad2}^{\text {mdf-2 }}$ (RNAi) embryos expressing GFP::PH and mCherry::Histone H2B to mark the plasma membrane and the chromosomes, respectively. The interval from NEBD to OCC (arrowheads) was measured. Scale bar $=10 \mu \mathrm{m}$. (C) The mean NEBD to OCC interval for the indicated conditions is plotted for both the first $\left(\mathrm{P}_{0}\right.$, top $)$ and second $(\mathrm{AB}$, bottom $)$ mitotic divisions. Error bars are the $95 \%$ confidence interval. 
The delay in HCP-1/2-depleted embryos was abolished by Mad2 ${ }^{\mathrm{MDF}-2}$ or Mad3 ${ }^{\mathrm{SAN}-1}$ codepletion, but it was of lower magnitude compared with the delay induced by monopolar spindles (Figure 2C). Codepletion of ZYG-1 did not increase the delay resulting from $\mathrm{HCP}-1 / 2$ depletion, indicating that in addition to performing a function that prevents checkpoint activation, $\mathrm{HCP}-1 / 2$ also make a positive contribution that increases the magnitude of the checkpoint signal.

In addition to the systematic analysis of kinetochore proteins described above, we also analyzed whether the inner centromere-localized Aurora B BIR-2 kinase subunit of the chromosomal passenger complex or the putative single Shugoshin family protein SGO-1 in C. elegans (C33H5.15; Kitajima et al., 2005) are required for checkpoint signaling. We did not observe abrogation of the monopolar-spindle induced cell cycle delay after inactivation of Aurora B ${ }^{\text {AIR-2 }}$ by using a temperature-sensitive mutant allele (or707ts; Severson et al., 2000; Supplemental Figure S2A) or after sgo1(RNAi) (Supplemental Figure S2B).

When considered in light of the assembly hierarchy of the kinetochore (Figure 2A), the above-mentioned data confirm that checkpoint signaling requires a core kinetochore scaffold. In addition, the results suggest that recruitment of three different components (the NDC-80 complex, the RZZ complex, and BUB-1) by KNL-1 is critical for checkpoint activation.

\section{Checkpoint Signaling Status after Inhibition of the Three Classes of Kinetochore Constituents Correlates with GFP::Mad2 ${ }^{M D F-2}$ Enrichment at Unattached Kinetochores}

Checkpoint activation correlates with the enrichment of specific components of the pathway, most prominently Mad2, on unattached kinetochores (Musacchio and Salmon, 2007). This enrichment is thought to reflect the local kinetochorecatalyzed reaction that generates the inhibitor of the APC/C. To correlate Mad2 recruitment with the functional analysis of checkpoint signaling, we generated a strain stably coexpressing GFP::Mad2 ${ }^{\mathrm{MDF}-2}$ and mCherry-Histone H2b. In the early mitotic divisions of control embryos, GFP::Mad2 ${ }^{\mathrm{MDF}-2}$ fluorescence is detected at the nuclear envelope/nucleoplasm beginning in prophase. After NEBD, GFP::Mad2 ${ }^{\mathrm{MDF}-2}$ remains present as a "cloud" of diffuse fluorescence surrounding the chromatin until anaphase onset, at which point it rapidly dissipates (Figure 3A and Supplemental Movie S5). Thus, no significant kinetochore localization of GFP::Mad2 ${ }^{\mathrm{MDF}-2}$ is observed in control embryos. In embryos depleted of ZYG-1 or SAS-6, GFP::Mad2 ${ }^{\mathrm{MDF}-2}$ localization was indistinguishable from controls during the first bipolar mitotic division (data not shown). However, during the second monopolar division, GFP::Mad2 ${ }^{\mathrm{MDF}-2}$ accumulated on the away-from-pole side of the chromatin after NEBD, reaching its peak intensity within 2 min (Figure 3, A and C, and Supplemental Movie S5) followed by decay of the signal. Thus, the accumulation of GFP::Mad2MDF-2 at kinetochores correlates with functional checkpoint signaling.

Immunoblotting indicated that the GFP::Mad2 ${ }^{\mathrm{MDF}-2}$ transgene was expressed at $\sim 1.5$ times the level of endogenous Mad2 ${ }^{\mathrm{MDF}-2}$ (Figure 3B) and that it caused a monopolar spindle-induced delay in the Mad2 $2^{\mathrm{MDF}-2}$ deletion strain $m d f$ 2(tm2190) (Supplemental Figure S3A). GFP::Mad2MDF-2 localization was qualitatively similar on monopolar spindles generated in the deletion mutant strain. We also observed partial rescue of the variable and low brood size phenotype of the $m d f-2(t m 2190)$ strain (data not shown). Because the transgene is expressed under the pie-1 promoter (Green et al., 2008), a lack of full rescue may reflect restricted expression.
We next analyzed the recruitment of GFP::Mad2 ${ }^{\mathrm{MDF}-2}$ to unattached kinetochores after depletion of the three classes of kinetochore components (Figure 3D). GFP::Mad2 ${ }^{\mathrm{MDF}-2}$ failed to accumulate on monopolar spindle-associated chromosomes after depletion of class I components, which are essential for checkpoint signaling. By contrast, depletion of class II components, which are not required for the monopolar spindle induced delay, did not affect the kinetochore accumulation of GFP::Mad2 ${ }^{\mathrm{MDF}-2}$. AuroraB ${ }^{\mathrm{AIR}-2}$ inhibition, which does not abrogate the checkpoint-induced delay, also did not affect kinetochore accumulation of GFP::Mad2 ${ }^{\text {MDF-2 }}$ (Supplemental Figure S2C). Consistent with the fact that their depletion triggers the checkpoint even in the absence of monopolar spindles, depletion of the class III components HCP-1/2 induced GFP::Mad2 ${ }^{\mathrm{MDF}-2}$ accumulation both in the presence and absence of monopolar spindles (Figure 3D). These results support a strict correlation between the ability of unattached kinetochores to induce a cell cycle delay and their ability to recruit GFP::Mad2 ${ }^{\mathrm{MDF}-2}$, providing strong support for the model that the kinetochore scaffoldbased local recruitment of $\mathrm{Mad} 2^{\mathrm{MDF}-2}$ is required to generate the signal that inhibits APC/C activity.

\section{GFP::Mad2 $2^{M D F-2}$ Accumulation at Kinetochores Is Unaffected By Depletion of Mad3 ${ }^{S A N-1}$ and Is Reduced, but Not Eliminated, by Depletion of BUB-3}

We next investigated GFP-Mad2 ${ }^{\mathrm{MDF}-2}$ localization at unattached kinetochores after depletion of conserved checkpoint pathway proteins (Figure 1D). We expected that because all of these proteins are required for the monopolar spindleinduced delay (Figure 1E), their depletion would eliminate GFP::Mad2 ${ }^{\mathrm{MDF}-2}$ localization, as observed for class I kinetochore components. This was indeed the case after depletion of Mad1 ${ }^{\mathrm{MDF}-1}$, BUB-1, or ROD-1 (Figure 3E). However, depletion of Mad3 ${ }^{\mathrm{SAN}-1}$ had no significant effect on GFP::Mad2MDF-2 localization at unattached kinetochores (Figure 3E, Supplemental Figure S3B, and Supplemental Movie S8). To confirm this result, we repeated the analysis using a viable null mutant of san-1 (san-1(ok1580); referred to subsequently as $\mathrm{Mad}^{\mathrm{san}^{\mathrm{s}} \mathrm{A}} \Delta$ ) that, similar to Mad3 ${ }^{\mathrm{SAN}-1}$ depletion by RNAi, is unable to generate a monopolar spindleinduced cell cycle delay (Supplemental Figure S4A). Even in the $\mathrm{Mad}^{\operatorname{san}^{-1}} \Delta$ strain, we did not see a significant reduction in the accumulation of GFP::Mad2 ${ }^{\mathrm{MDF}-2}$ at unattached kinetochores compared with controls (Figure 3F and Supplemental Movie S9). Depletion of BUB-3 reduced the accumulation of GFP::Mad2 ${ }^{\mathrm{MDF}-2}$ but did not eliminate its kinetochore localization (Figure 3E and Supplemental Movie S7). Quantitative analysis of the peak GFP::Mad2 ${ }^{\mathrm{MDF}-2}$ fluorescence on chromosomes of monopolar spindles confirmed these observations (Supplemental Figure S3B). We conclude that Mad3 ${ }^{\mathrm{SAN}-1}$ and BUB-3 are not essential for the accumulation of GFP::Mad2 ${ }^{\mathrm{MDF}-2}$ at unattached kinetochores.

\section{Mad3 ${ }^{\text {SAN-1 }}$ Does Not Enrich at Unattached Kinetochores When the Spindle Checkpoint Is Active}

Mad3 $3^{\mathrm{SAN}-1}$ is not required for Mad2 $2^{\mathrm{MDF}-2}$ to accumulate at unattached kinetochores. To determine whether the converse is also true, we generated a strain coexpressing mCherry-Histone H2b and GFP::Mad3 $3^{\mathrm{SAN}-1}$. Expression of the Mad3 $3^{\mathrm{SAN}-1}$ transgene restored a monopolar spindle-induced cell cycle delay in the Mad3 ${ }^{\text {san-1}} \Delta$ strain (Supplemental Figure S4A). In control embryos, GFP::Mad3 ${ }^{\text {SAN-1 }}$ showed diffuse localization in the vicinity of chromatin at prometaphase, which seemed significantly reduced by metaphase; there was no signal above background in other stages of mitosis (Figure 4A and Supplemental Movie S10). Sur- 
A

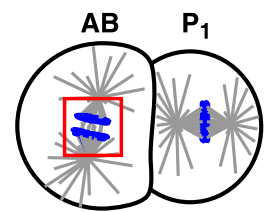

\section{Control}

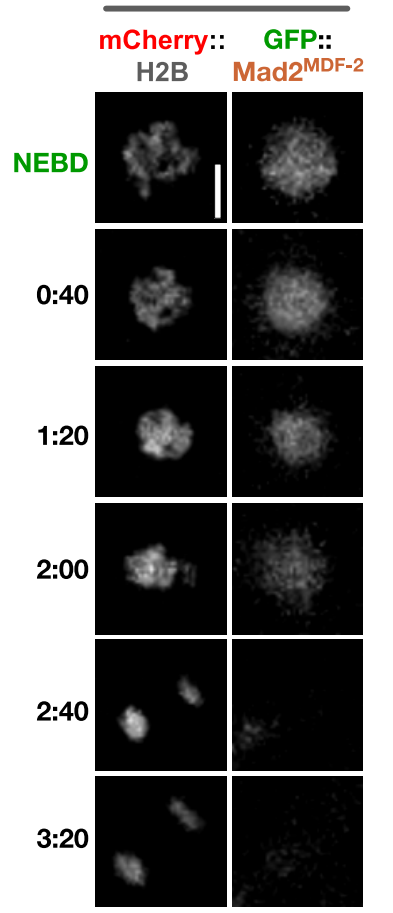

B

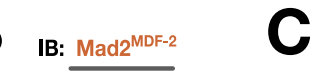
97- $\overline{\text { N2 OD110 }}$

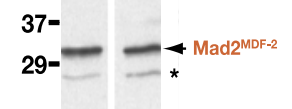

20-

53- $-\frac{\text { IB: }}{+\alpha \text {-tubulin }}$
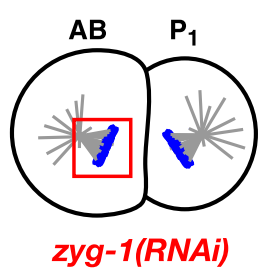

mCherry:: GFP:: H2B Mad2 ${ }^{\text {MDF-2 }}$
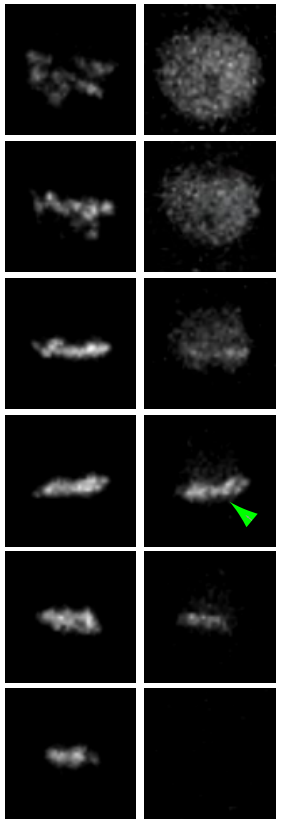

.
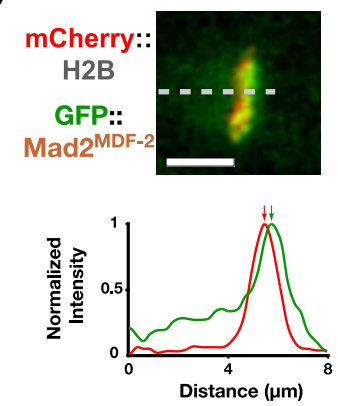

$\mathbf{F}$

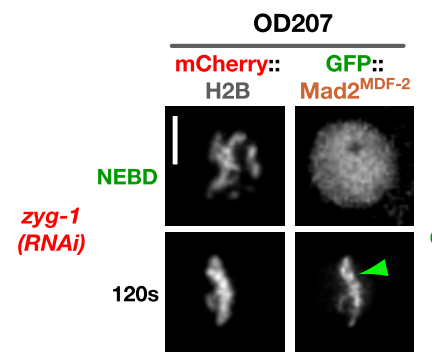

D

\section{NEBD}

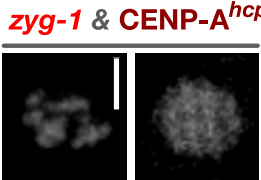

$120 \mathrm{~s}$
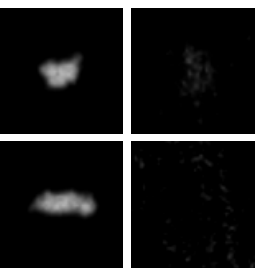

240s
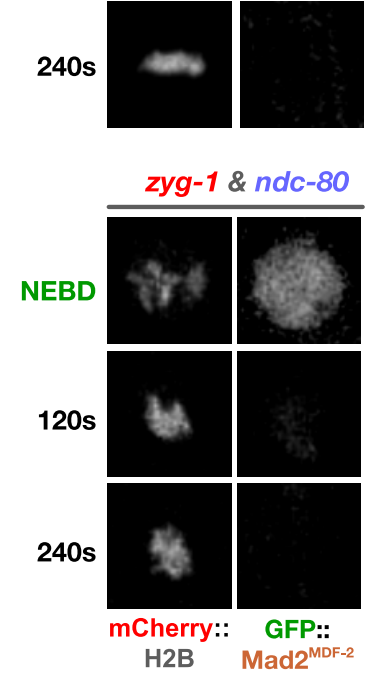

E
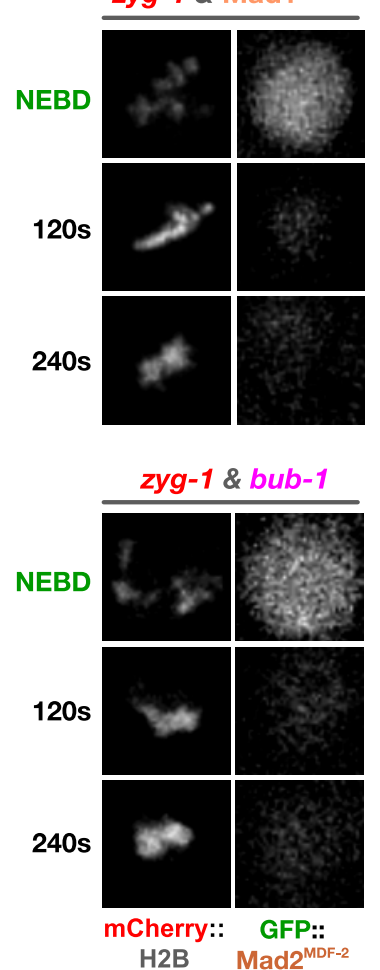

zyg-1 \& CENP-C ${ }^{\text {hcp-4 }}$
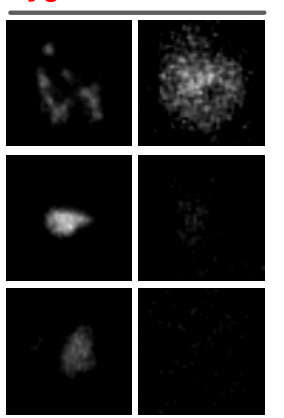

zyg-1 \& MCAK M/p-7 $^{k / p}$
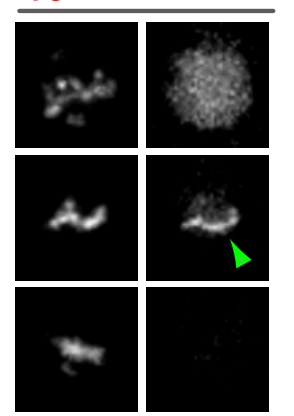

mCherry:: GFP::

H2B Mad2 ${ }^{\text {MDF-2 }}$
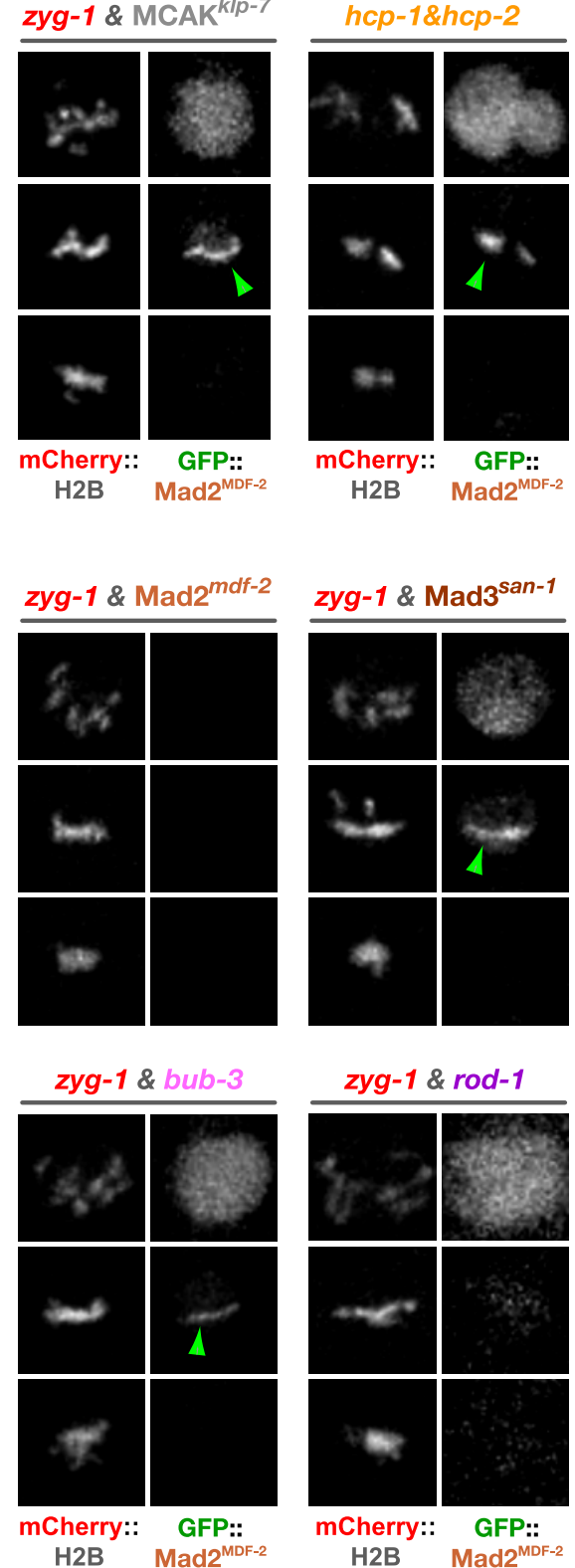

mCherry:: GFP:: H2B Mad2 ${ }^{\text {MDF-2 }}$

zyg-1 \& knl-1
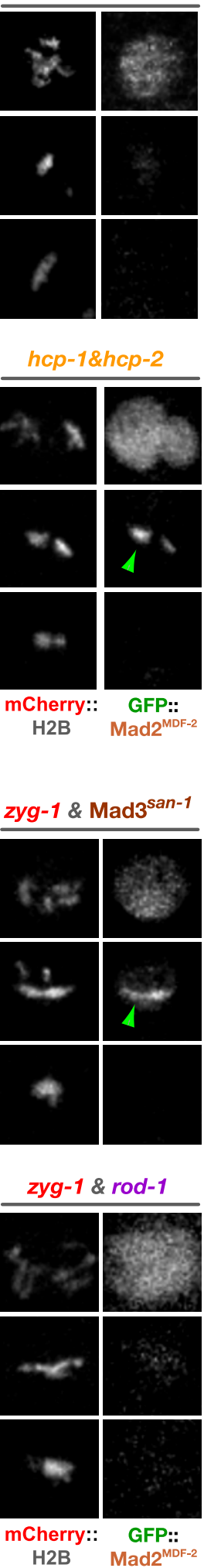

Figure 3. The accumulation of GFP::Mad2 ${ }^{\mathrm{MDF}-2}$ on chromosomes associated with monopolar spindles correlates with checkpoint activation (A) Selected frames from time-lapse sequences of embryos expressing GFP::Mad2 ${ }^{\mathrm{MDF}-2}$ and mCherry::H2B that have normal bipolar spindles (control, left) or monopolar spindles [zyg-1(RNAi), right]. Images correspond to boxed regions of the AB cell depicted in the schematics. GFP-MDF-2 ${ }^{\text {MDF-2 }}$ fluorescence accumulates on chromosomes associated with monopolar spindles (green arrowhead). Bar, $5 \mu \mathrm{m}$. (B) A Western blot of N2 (wild-type) and OD110 (coexpressing mCherry::H2b and GFP::Mad2 ${ }^{\mathrm{MDF}-2}$ ) strains was probed with an anti::Mad2 ${ }^{\mathrm{MDF}-2}$ antibody. The Mad2 ${ }^{\mathrm{MDF}-2}$ and GFP::Mad2 ${ }^{\mathrm{MDF}-2}$ bands are indicated; asterisks mark nonspecific bands that are not eliminated by Mad2 ${ }^{\mathrm{MDF}-2}$ RNAi. The blot was also probed with an antibody to $\alpha$-tubulin as a loading control. (C) GFP::Mad-2 ${ }^{\mathrm{MDF}-2}$ accumulates asymmetrically on the 
prisingly, we did not detect enrichment of GFP::Mad3 $3^{\text {SAN-1 }}$ at kinetochores of monopolar spindle-associated chromosomes; instead, we observed a diffuse localization pattern similar to that in control embryos with bipolar spindles (Figure 4B and Supplemental Movie S10). This localization pattern was unchanged in the absence of a wild-type Mad3 ${ }^{\text {SAN-1 }}$ allele (Figure 4C and Supplemental Movie S11) and was eliminated by RNAi-mediated depletion of Mad3 $3^{\text {SAN-1 }}$ (Supplemental Figure S4B). Codepletion of Mad2 ${ }^{\mathrm{MDF}-2}$, Mad1 ${ }^{\mathrm{MDF}-1}$, or BUB-1 had no significant effect on this diffuse localization; by contrast, in BUB-3-depleted embryos, the GFP signal was significantly diminished (Figure 4B and Supplemental Movies S12 and S13). The latter observation suggests that $\mathrm{Mad}^{\mathrm{SAN}-1}$ protein may be destabilized after depletion of BUB-3; we were unable to confirm this due to lack of a suitable anti-Mad3 $3^{\mathrm{SAN}-1}$ antibody. We conclude that Mad3 $3^{\mathrm{SAN}-1}$ is not coenriched on unattached kinetochores and that its stability may be dependent on BUB-3.

\section{BUB-3 Exhibits Basal Kinetochore Localization}

That Is Enriched at Unattached Kinetochores in a BUB-1dependent but Mad1 $1^{M D F-1} / M a d 2^{M D F-2}$-independent Manner

We next generated a strain coexpressing GFP::BUB-3 and mCherry-Histone $\mathrm{H} 2 \mathrm{~b}$ and performed experiments similar to those performed for Mad3 $3^{\mathrm{SAN}-1}$. Both endogenous BUB::3 (Supplemental Figure S5A) and GFP::BUB-3 (Figure 4D) were detected at kinetochores of control embryos. BUB::3 is first detectable on condensing chromosomes in late prophase and reaches maximal fluorescence intensity as paired lines on kinetochores at metaphase (Figure 4D and Supplemental Movie S14). It rapidly dissipates from kinetochores in early anaphase and is no longer detectable by late anaphase/early telophase. In cells with monopolar spindles, GFP::BUB-3 becomes enriched on the unattached kinetochores on the chromosomal face away from the pole (Figure $4 \mathrm{E}$ and Supplemental Movie S14). We conclude that BUB-3 has a basal kinetochore localization that is amplified when the checkpoint is active.

We next wanted to investigate the relationship between BUB-3 enrichment and Mad2 ${ }^{\mathrm{MDF}-2}$ enrichment at unattached kinetochores. We did not observe an effect of depleting either Mad1 ${ }^{\mathrm{MDF}-1}$ or Mad2 ${ }^{\mathrm{MDF}-2}$ on the enrichment of BUB-3 at unattached kinetochores (Figure 4E). We also did not observe an effect of depleting Mad3 $3^{\text {SAN-1 }}$ (Figure 4E), indicating that BUB-3 levels and localization are independent of Mad3 ${ }^{\mathrm{SAN}-1}$. By contrast, depletion of BUB-1 eliminated BUB-3 localization on both control bipolar (data not shown) and monopolar spindles (Figure 4E and Supplemental Movie S15); depletion of the RZZ complex subunit ROD-1, reduced the level of BUB-3 at unattached kinetochores, although localization was still evident (Figure 4E

Figure 3 (cont). chromosomal surface pointing away from the single spindle pole in monopolar spindles. The line scan (5-pixel wide; normalized relative to maximum intensity in each channel) illustrates the asymmetric distribution. Bar, $5 \mu \mathrm{m}$. (D and E) Selected frames from time-lapse sequences of GFP::Mad2 ${ }^{\mathrm{MDF}-2}$;mCherry::H2B strain after the indicated perturbations of kinetochore proteins (D) and conserved checkpoint proteins $(E)$. Note GFP::Mad2 ${ }^{\mathrm{MDF}-2}$ accumulation at kinetochores (green arrowheads) in $z y g-1+\mathrm{MCAK}^{k l p-7}(R N A i)$ and in $h c p-1 / 2$ (RNAi) in D and in zyg-1+Mad3 $3^{\text {san }-1}$ (RNAi) and $z y g-1+b u b-3$ (RNAi) in E. Bar, $5 \mu \mathrm{m}$. (F) Selected frames from a time-lapse sequence of a monopolar spindle (AB cell) in the $\mathrm{Mad}^{\mathrm{san}-1} \Delta$ mutant strain into which the GFP::Mad2 $2^{\mathrm{MDF}-2}$ transgene was introduced by mating. Note accumulation of Mad2 ${ }^{\mathrm{MDF}-2}$ at kinetochores (green arrowhead). Bar, $5 \mu \mathrm{m}$. and Supplemental Movie S16), but depletion of the Ndc80 complex did not have a significant effect (Supplemental Figure S5C).

In converse experiments, BUB-3 depletion had no effect on BUB-1 (Figure 4F) or RZZ complex kinetochore localization (data not shown). Because BUB-3 depletion does not lead to embryonic lethality, whereas depletion of BUB-1 or ROD-1 leads to penetrant lethality, these results suggest that BUB-3 is not essential for the other chromosome segregation functions of BUB-1 and the RZZ complex. We conclude that BUB-3 exhibits basal kinetochore localization and accumulates at checkpoint signaling kinetochores in a BUB-1-dependent manner.

\section{The NDC-80 Complex, the RZZ Complex, and BUB-1 Converge Downstream of KNL-1 to Direct the Accumulation of Mad2 $2^{M D-2}$ and BUB-3 and Checkpoint Activation}

The NDC-80 complex, BUB-1, and the RZZ complex are all dependent on KNL-1 for their kinetochore localization (Desai et al., 2003; Cheeseman et al., 2004) and are all essential for checkpoint activation. Previous work has shown that NDC-80 complex is recruited to kinetochores independently of BUB-1 and the RZZ complex (Desai et al., 2003; Gassmann et al., 2008). Consistent with this, localization of BUB-3, which depends on BUB-1, is independent of the NDC-80 complex (Supplemental Figure S5C). We extended this analysis to show that BUB-1 and the RZZ complex also target to kinetochores independently of each other (Figure 4G). Thus, three components with distinct functions that are independently targeted to kinetochores by KNL-1 are integrated to direct Mad2 ${ }^{\mathrm{MDF}-2}$ and BUB-3 recruitment and checkpoint activation (Figure $4 \mathrm{H}$ ). Interestingly, the kinetochore targeting of Mad2 $2^{\mathrm{MDF}-2}$ and BUB-3 reflect different, largely independent, pathways downstream of NDC-80, BUB-1, and the RZZ complex (Figure 4H). The kinetochore accumulation of Mad2 ${ }^{\mathrm{MDF}-2}$ (and presumably also Mad1 ${ }^{\mathrm{MDF}-1}$ ) requires NDC-80, BUB-1, and the RZZ complex and is enhanced by (but does not require) BUB-3. The kinetochore localization of BUB-3 requires BUB-1 and is enhanced by the presence of the RZZ complex, but it does not require Mad1 $1^{\mathrm{MDF}-1}$ or Mad2 ${ }^{\mathrm{MDF}-2}$. The existence of distinct pathways for the recruitment of Mad2 ${ }^{\mathrm{MDF}-2}$ and $\mathrm{BUB}-3$ may facilitate the integration of different inputs during spindle checkpoint activation.

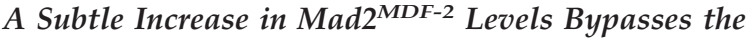 Requirement for Mad3 $3^{\text {SAN-1 }}$ and BUB-3 to Elicit a Kinetochore-dependent Monopolar Spindle-induced Cell Cycle Delay}

In the strain expressing both endogenous and GFP::Mad2MDF-2, basal cell cycle timing was unaffected and monopolar spindles increased the NEBD-DCON interval (Figure 5A). Because this increase was dependent on Mad1 ${ }^{\mathrm{MDF}-1}$ (Figure 5A) and KNL-1 (data not shown), it reflects kinetochoredependent signaling and excludes the trivial possibility that overexpression of Mad2 $2^{\mathrm{MDF}-2}$ is causing a cell cycle delay by general cytoplasmic inhibition of the APC/C. Strikingly, depletion of Mad3 $3^{\text {SAN-1 }}$ or BUB-3 did not eliminate the monopolar spindle-induced delay in this strain (Figure 5A). The same result was obtained after crossing the GFP::Mad2 ${ }^{\mathrm{MDF}-2}$ transgene into the Mad3 ${ }^{\text {san-1}} \Delta$ strain background (Figure 5B). Importantly, the monopolar spindle-induced delay in the $\mathrm{Mad}^{\mathrm{san}^{-1}} \Delta$ strain expressing the GFP::Mad2 ${ }^{\mathrm{MDF}-2}$ transgene required Mad1 ${ }^{\mathrm{MDF}-1}$ (Figure 5B), indicating that the Mad3 $3^{\mathrm{SAN}-1}$-independent delay was kineto- 
A

Figure 4. Analysis of Mad3 $3^{\mathrm{SAN}-1}$ and BUB-3 localization in control and checkpoint-activated embryos. (A and B) Selected frames from timelapse sequences of embryos coexpressing mCherry::Histone $\mathrm{H} 2 \mathrm{~b}$ and GFP::Mad3 $3^{\text {SAN-1 }}$ are shown for the indicated conditions. GFP::Mad3 ${ }^{\text {SAN-1 }}$ is diffusely localized in the nuclear area in control embryos (A) but does not accumulate at unattached kinetochores of monopolar spindles (zyg-1(RNAi) in $\mathrm{B}$; in addition, fluorescence levels of GFP::Mad ${ }^{\mathrm{SAN}-1}$ are significantly reduced by depletion of BUB-3 [compare with Mad3 $^{\text {san-1 }}$ (RNAi) shown in Supplemental Figure S4B). Bars, $5 \mu \mathrm{m}$. (C) GFP::Mad3 ${ }^{\text {SAN-1 }}$ fails to accumulate at kinetochores of monopolar spindles even when endogenous Mad3 ${ }^{S A N-1}$ is absent. Selected frames from a Mad3 $3^{\text {san-1}} \Delta$ mutant embryo into which the GFP::Mad3 $3^{\text {SAN-1 }}$ transgene was introduced by mating. Bar, $5 \mu \mathrm{m}$. (D and E) Selected frames from time-lapse sequences of embryos coexpressing mCherry::Histone $\mathrm{H} 2 \mathrm{~b}$ and GFP::BUB-3 are shown for the indicated conditions. Unlike GFP:: Mad2 ${ }^{\mathrm{MDF}-2}$ and GFP::Mad3 ${ }^{\mathrm{SAN}-1}$, GFP::BUB-3 is detected at kinetochores of bipolar spindles of control embryos. GFP::BUB-3 additionally accumulates on unattached kinetochores associated with monopolar spindles [zyg-1(RNAi) in E]. GFP::BUB-3 localization to kinetochores depends on BUB-1 but is independent of Mad1 ${ }^{\text {MDF-1, }}$, Mad2 ${ }^{\mathrm{MDF}-2}$, or Mad3 ${ }^{\mathrm{SAN}-1}$; depletion of ROD-1, a subunit of the RZZ complex, reduces BUB-3 accumulation on unattached kinetochores. Bars, $5 \mu \mathrm{m}$. (F) BUB-3 depletion does not perturb kinetochore localization of BUB-1. The efficacy of the BUB-3 depletion was established using immunofluorescence with an anti-BUB-3 antibody (Supplemental Figure S5). Bar, $10 \mu \mathrm{m}$. (G) BUB-1 and the RZZ complex target independently of each other to the kinetochore. Bar, $10 \mu \mathrm{m}$. (H) Summary of the relationships between outer kinetochore components and Mad2 ${ }^{\mathrm{MDF}-2}$ and BUB-3 localization at checkpoint signaling kinetochores.

D
E

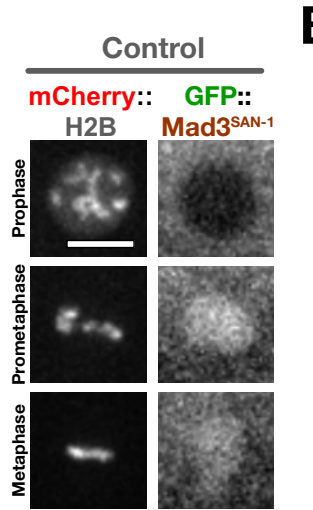

B

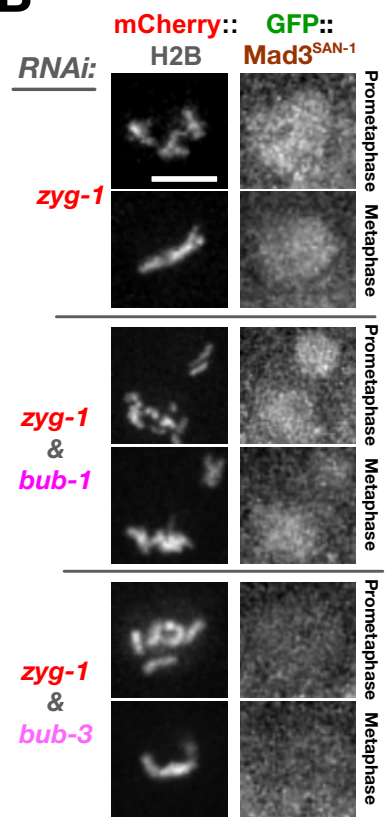

Mad3 $3^{5}: 12$

GFP::Mad3 ${ }^{\text {SAN-1 }}$
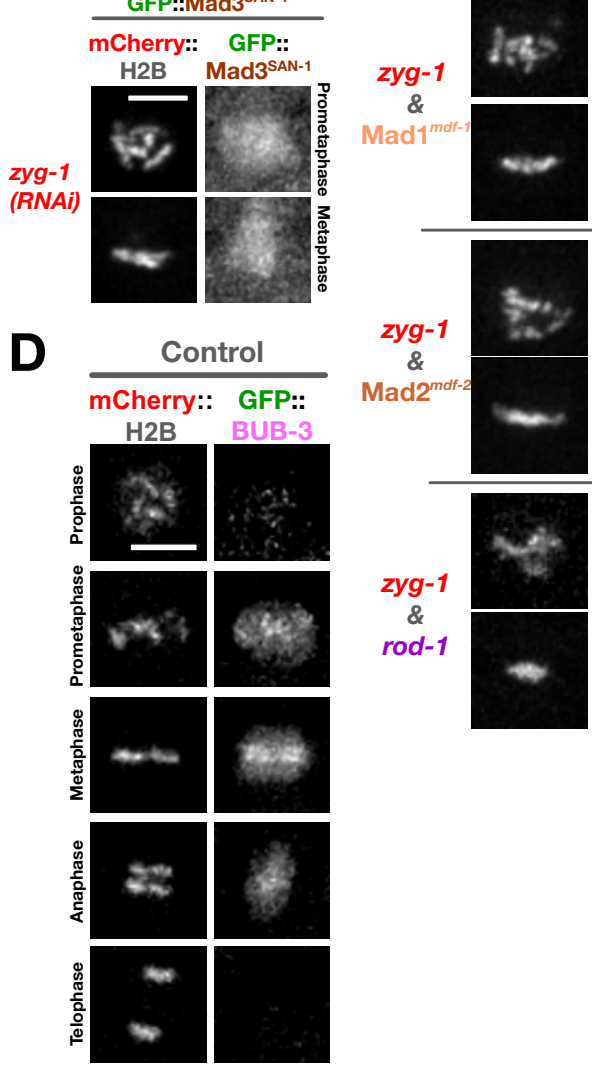

G

H
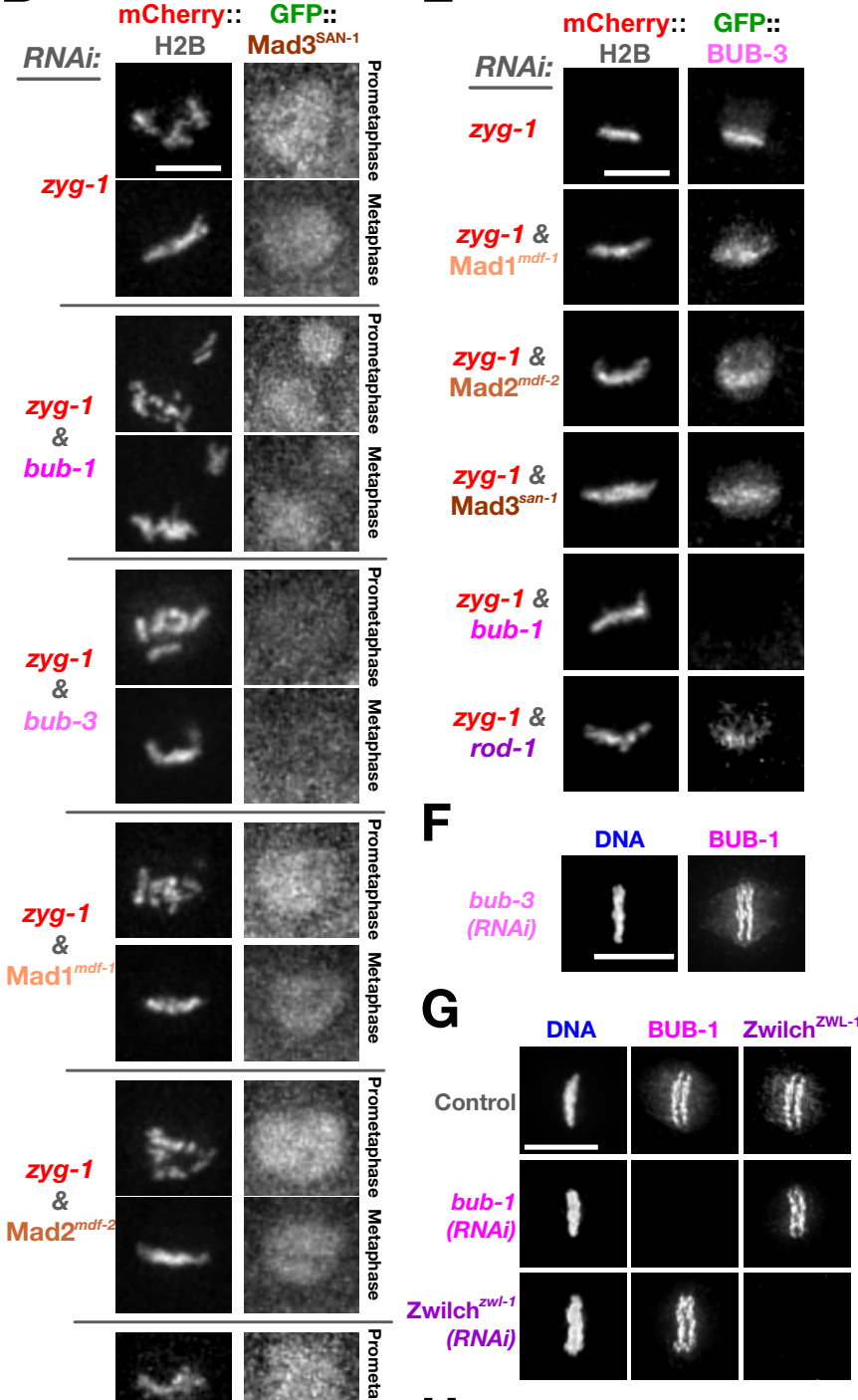

Kinetochore:

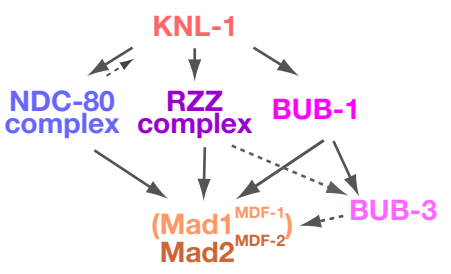

Cytoplasm:

BUB-3 $\longrightarrow$ Mad3 $^{\text {SAN-1 }}$

(protein stability?) chore dependent. We did not observe a bypass of the requirement for Mad1 ${ }^{\mathrm{MDF}-1}$ in the strain expressing GFP::BUB-3 (Supplemental Figure S5D), indicating that kinetochore-localized Mad1 $1^{\mathrm{MDF}-1} / \mathrm{Mad}^{\mathrm{MDF}-2}$ is indispensable for checkpoint signaling and that the bypass only works one-way.

Because GFP::Mad2 ${ }^{\mathrm{MDF}-2}$ was expressed from the transgene at 1.5 times the level of endogenous Mad2 ${ }^{\mathrm{MDF}-2}$ (Figure
$3 \mathrm{~B})$, the total level of Mad2 ${ }^{\mathrm{MDF}-2}$ in the GFP::Mad2 $2^{\mathrm{MDF}-2}$ strain was $\sim 2.5$ times that in controls. These results suggest that a subtle increase in Mad2MDF-2 levels is sufficient to bypass the requirement for $\mathrm{Mad}^{\mathrm{SAN}-1}$ or BUB-3 to elicit a monopolar spindle-induced kinetochore-dependent cell cycle delay. If this were true, then restoring Mad2 ${ }^{\mathrm{MDF}-2}$ expression to endogenous levels should reverse this effect. To test this prediction, we used dsRNAs 


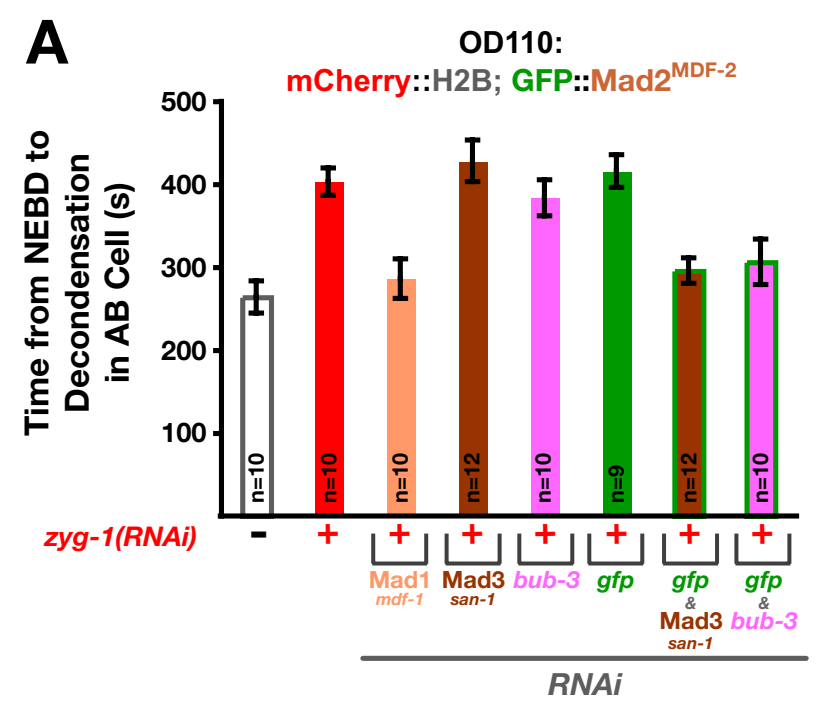

B

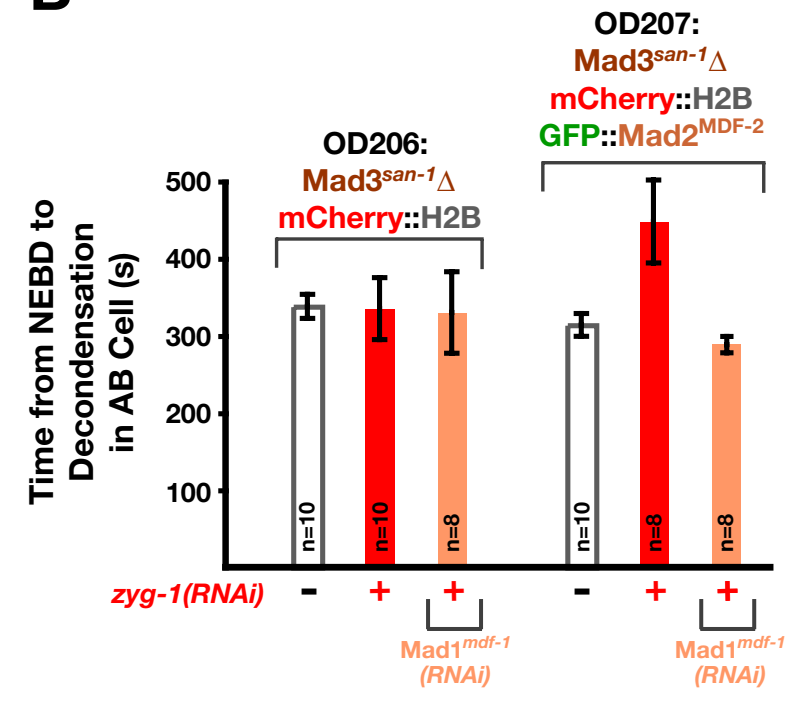

targeting GFP and ZYG-1 to simultaneously eliminate expression of the GFP-Mad2 ${ }^{\mathrm{MDF}-2}$ transgene and generate monopolar spindles. In this condition, a delay in the NEBD-DCON interval was observed that was not significantly different from ZYG-1 depletions alone (Figure 5A); the lack of any GFP signal on the chromosomes confirmed the efficacy of the GFP dsRNA (Figure 5C). When we then additionally codepleted Mad3 $3^{\text {SAN-1 }}$ or BUB-3, the monopolar spindle-induced delay in the NEBD-DCON interval was eliminated, indicating that the bypass of the requirement for $\mathrm{Mad} 3^{\mathrm{SAN}-1}$ and BUB-3 is dependent on the expression of the GFP-Mad2 $2^{\mathrm{MDF}-2}$ transgene (Figure 5A).

It is possible that the GFP::Mad2 $2^{\mathrm{MDF}-2}$ fusion is functionally altered in terms of $\mathrm{APC} / \mathrm{C}$ inhibitory activity; however, neither basal cell cycle timing nor the extent of the kinetochore-dependent delay, both of which are sensitive to APC/C inhibition, were significantly affected by its presence. We conclude that a subtle increase in Mad2 ${ }^{\mathrm{MDF}-2}$ levels bypasses the requirement for Mad3 ${ }^{\text {SAN-1 }}$ and BUB-3 in kinetochore-dependent spindle checkpoint signaling.
C

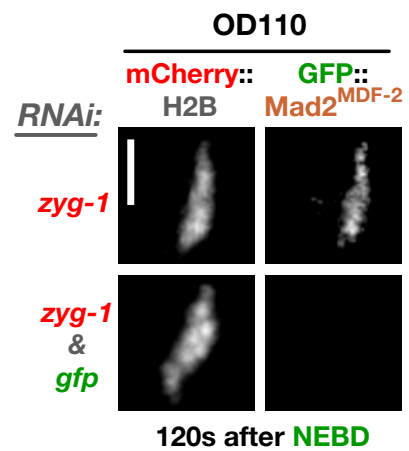

Figure 5. A subtle increase in Mad2 ${ }^{\mathrm{MDF}-2}$ levels bypasses the requirement for Mad3 $3^{\mathrm{SAN}-1}$ and BUB-3 in generating a monopolar spindleinduced cell cycle delay. (A and $\mathrm{B}$ ) The mean NEBD to DCON interval in the $\mathrm{AB}$ cell is plotted for the indicated conditions/strains. Error bars are the $95 \%$ confidence interval. (C) A selected frame (120 s after NEBD) from time-lapse sequences of the GFP::Mad2 ${ }^{\mathrm{MDF}-2}$ transgene-expressing strain injected with dsRNAs targeting $z y g-1$ alone (top row) or GFP and $z y g-1$ (bottom row). Bar, $5 \mu \mathrm{m}$.

\section{DISCUSSION}

Systematic Analysis of the Requirements for Spindle Checkpoint Activation Indicates a Central Role for the KMN Network

Here, we use controlled monopolar spindle formation to perform a systematic analysis of the requirements for checkpoint activation and Mad2 ${ }^{\mathrm{MDF}-2}$ recruitment in the C. elegans embryo. Our analysis comparing the classification of kinetochore proteins into functional groups based on phenotypic analysis and their position in the kinetochore assembly hierarchy to their role in checkpoint activation (Figure 6A), strongly supports the model that a kinetochore-triggered reaction is central to checkpoint activation. Specifically, all tested inhibitions that abrogate outer kinetochore assembly, including depletion of the centromeric histone CENP-A, prevented checkpoint activation. This result seems contradictory to checkpoint-dependent mitotic delays reported after inhibition of the centromeric histone CENP-A in Drosophila embryos and vertebrate cells (Regnier et al., 2005; Blower et al., 2006). It is possible that this reflects a difference in kinetochore assembly pathways between these systems. Al- 
A

Figure 6. Summary of the systematic analysis and a model for the bypass of Mad3 $3^{\text {SAN-1 }}$ and BUB-3 by a subtle increase in Mad2 ${ }^{\mathrm{MDF}-2} \mathrm{lev}-$ els. (A) Summary of the requirements for checkpoint activation \& GFP::Mad2 ${ }^{\text {MDF-2 }}$ accumulation at unattached kinetochores. Kinetochore components are placed into different functional groups based on prior in vivo and in vitro studies. The three classes of components, with respect to their role in checkpoint activation, are defined in the legend and are indicated by the colored boxes. (B) A model based on the Mad2 "template/conformational dimerization" model for why an increase in Mad2 $2^{\mathrm{MDF}-2}$ levels reduces the need for Mad3 $3^{\text {SAN-1 }}$ and BUB-3 to induce a kinetochoredependent cell cycle delay. For simplicity, free $\mathrm{Cdc} 20^{\mathrm{FZY}-1}$ molecules are not drawn. Left, wild-type is depicted, in which Mad2-C generated at the kinetochore is integrated with cytoplasmic Mad3 $3^{\text {SAN-1/BUB-3 to }}$ inhibit the APC/C. Right, an increased flux of Mad2 results in higher levels of Mad2-C and bypasses the requirement for Mad3 ${ }^{\text {SAN-1 }}$ / BUB-3.

B
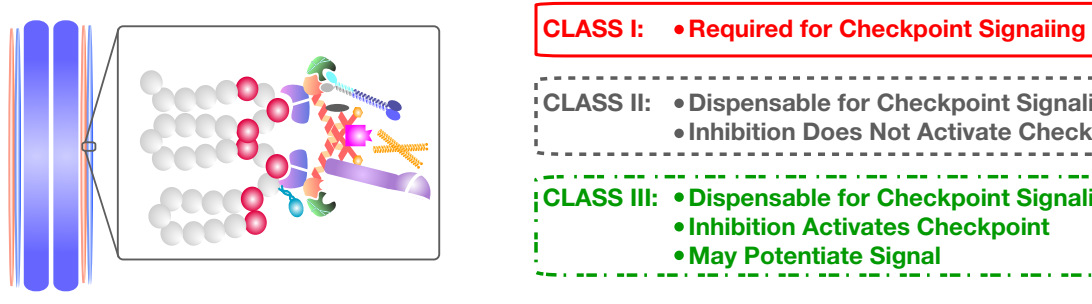

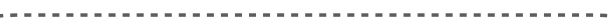
CLASS II: - Dispensable for Checkpoint Signaling - Inhibition Does Not Activate Checkpoint 'CLASS III: -Dispensable for Checkpoint Signaling ¡CLASS III: - Dispensable for Checkpoint Signaling - Inhibition Activates Checkpoint ! . May Potentiate Signal . . . . . . . . . . . .
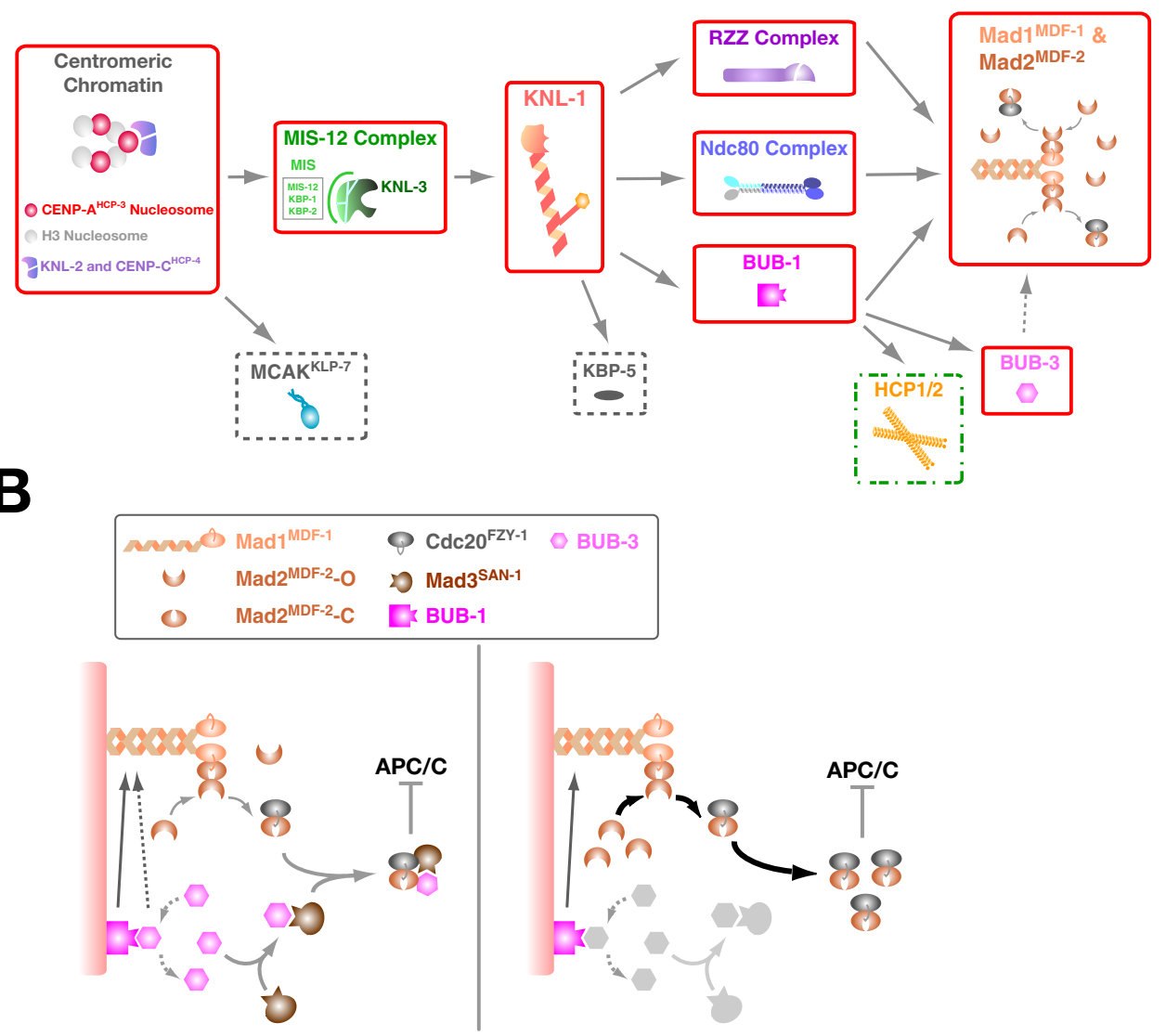

ternatively, the high stability of CENP-A, which does not affect intrinsic turnover-independent RNAi-mediated depletion in C. elegans (Oegema and Hyman, 2006) but does affect depletion efficacy in mutant Drosophila embryos that contain maternal product (Blower et al., 2006) or in chicken cells where expression of a rescuing transgene is turned off (Regnier et al., 2005), may account for the difference.

Our systematic analysis identifies the core microtubule binding site of the kinetochore, the KMN network, as the most downstream stably kinetochore-localized protein group required for checkpoint activation. Specifically, three components with distinct functions that are independently targeted to kinetochores by the scaffold protein KNL-1-the NDC-80 complex, the RZZ complex, and BUB-1-are all critical for checkpoint activation. The NDC-80 complex directly associates with KNL-1 in the KMN network (Cheeseman et al., 2004) and has been implicated in checkpoint signaling in other systems (McCleland et al., 2003; Gillett et al., 2004; Meraldi et al., 2004). Based on work in human cells, the association of KNL-1 with BUB-1 is also likely to be direct (Kiyomitsu et al., 2007). At least in C. elegans, where a Zwint-like intermediate protein bridging KNL-1 and the $\mathrm{RZZ}$ complex is not present, the RZZ complex may also directly associate with KNL-1. Together, these findings suggest an analogy to signaling networks in which different inputs integrated by scaffold proteins control signaling reactions. In the case of the spindle checkpoint, mechanical inputs from two independently targeted microtubule-binding activities of distinct functions, one resident in the Ndc80 complex and the second in the dynein/dynactin motor complex targeted by the RZZ complex, are likely integrated with BUB-1 in the context of the KNL-1 scaffold. Investigating the mechanism of integration will require developing a means to model the checkpoint reaction in vitro with a faithful facsimile of the activation base provided by the kinetochore-such an effort should be facilitated by the reconstitution of the C. elegans KMN network (Cheeseman et al., 2006).

\section{Core Checkpoint Pathway in C. elegans}

In C. elegans, the core checkpoint pathway is simplified relative to other metazoan systems-no Mps1-like kinase exists and a Mad3, instead of a BubR1-like protein is present. It is possible that this simplification is linked to weakening of the checkpoint to accommodate the large diffuse kinetochores on the holocentric chromosomes of this organism. At 
least in the second embryonic division, which is the focus of our work, monopolar spindles are only able to extend the mitotic phase of the cell cycle twofold. Alternatively, the relatively small magnitude of the delay at the two-cell stage may reflect the large cytoplasm-to-nuclear ratio in the blastomeres at two-cell stage, consistent with the previously established relationship between the checkpoint signal efficacy and the nuclear-cytoplasmic ratio observed in Xenopus embryos (Minshull et al., 1994). A fast-acting temperaturesensitive mutant that permits generation of monopolar spindles in later embryonic cell divisions, in which the cells are smaller, should help distinguish between these possibilities in future work.

Depletion of the core checkpoint proteins had no effect on basal cell cycle timing, but all were essential for the monopolar spindle-induced cell cycle delay. In addition, recruitment of Mad2 $2^{\mathrm{MDF}-2}$ to kinetochores was observed only when the checkpoint was activated-no significant accumulation at kinetochores was evident in control embryos. By contrast, both BUB-1 and BUB-3 localized to kinetochores even without checkpoint activation. This is consistent with the idea that BUB-1 provides an essential function in chromosome segregation that is required for embryonic viability. These results are generally analogous to what has been reported in budding yeast, in whichMad1 and Mad2 localization is only observed after drug-induced microtubule depolymerization and where Bub1 and Bub3 mutants are significantly more sick than Mad1 and Mad2 mutants (Warren et al., 2002; Gillett et al., 2004). Several noncheckpoint functions for Bub1 family kinases have been reported in yeast and vertebrates (Johnson et al., 2004; Tang et al., 2004b; Kitajima et al., 2005; Vaur et al., 2005; Boyarchuk et al., 2007), and at least one of these functions (targeting of CENP-F-like proteins HCP-1/2 to kinetochores) is conserved in C. elegans embryos (Encalada et al., 2005; data not shown).

In addition to the core checkpoint proteins and the KMN network, we also observed a positive contribution to checkpoint signaling from HCP-1/2. Depletion of these proteins in cells with either bipolar or monopolar spindles triggers a Mad2 ${ }^{\mathrm{MDF}-2} / \mathrm{Mad}^{\mathrm{SAN}-1}$-dependent cell cycle delay, but the magnitude of this delay is less than that when HCP-1/2 are present. Synthetic genetic screens have identified HCP-1, but not HCP-2, as a contributor to checkpoint signaling in $C$. elegans (Tarailo et al., 2007; Hajeri et al., 2008)—our results extend these studies by showing that HCP-1/2 are not required for Mad2 ${ }^{\mathrm{MDF}-2}$ enrichment at kinetochores; HCP-1/2 may control the extent of Mad2 $2^{\mathrm{MDF}-2}$ accumulation or they may act at a different step that affects the potency of the inhibitory signal. Analogous conclusions have been made from studies on vertebrate CENP-F (for discussion, see Tarailo et al., 2007; Hajeri et al., 2008). Finally, MCAK ${ }^{\mathrm{KLP}-7}$ was dispensable for both checkpoint activation and Mad2MDF-2 kinetochore localization. This result is in contrast to a previous report that MCAK ${ }^{\mathrm{KLP}-7}$ is required for the checkpoint based on differential interference-contrast imaging of nocodazole-treated embryos (Encalada et al., 2005). The reason for this discrepancy is currently unclear; we note that inhibition of kinesin-13s in vertebrates has not suggested an involvement in checkpoint activation (e.g., see (Manning et al., 2007).

\section{Mad3 Versus BubR1 in the Core Checkpoint Pathway}

C. elegans is the only metazoan analyzed to date that lacks a BubR1-like kinase and instead has a truncated Mad3-like protein. An interesting emerging pattern is that the presence of a BubR1-like kinase correlates with the presence of a CENP-E-like kinetochore-localized kinesin motor (Chan et al., 1999; Abrieu et al., 2000). Worms and fungi, which have Mad3 instead of BubR1, lack CENP-E. The described functional links between CENP-E and the BubR1 kinase during checkpoint signaling in vertebrates are consistent with this pattern (Mao et al., 2005).

The most significant difference between Mad3 $3^{\mathrm{SAN}-1}$ in $C$. elegans and BubR1 in other metazoans is with respect to kinetochore localization. The BubR1-like proteins in Drosophila and vertebrates localize to kinetochores, whereas we find that a functional C. elegans GFP:Mad3 ${ }^{\text {SAN-1 }}$ does not. Interestingly, chromatin immunoprecipitation and microscopy failed to detect budding yeast Mad3 at kinetochores under spindle depolymerization conditions that significantly enriched Mad1 and Mad2 at kinetochores (Gillett et al., 2004). This similarity suggests that Mad3-like proteins, compared with BubR1-like protein kinases, are not enriched at kinetochores and, by inference, act primarily in the cytoplasm/nucleoplasm. However, contrary to this suggestion, fission yeast Mad3 localizes to kinetochores (Millband and Hardwick, 2002). Experiments in which the Mad3s are switched between the two yeasts and C. elegans may help define the signals that control Mad3 localization and elucidate its site of action with respect to checkpoint signaling. Whether the kinetochore localization of Mad3 in fission yeast or BubR1 in vertebrate cells is essential for checkpoint signaling has not been established. Recent studies in vertebrates are leading to the conclusion that, similar to our findings in C. elegans for Mad3 $3^{\mathrm{SAN}-1}$, the checkpoint signaling function of BubR1 is independent of kinetochores (Kulukian and Cleveland, personal communication); the kinetochore localization of BubR1 may contribute to a distinct noncheckpoint role in chromosome segregation (Lampson and Kapoor, 2005).

In C. elegans, Mad3 $3^{\mathrm{SAN}-1}$ and Mad2 ${ }^{\mathrm{MDF}-2}$ are both required for the monopolar spindle-induced cell cycle delay in the early embryo. However, that subtle overexpression of Mad2MDF-2 bypasses the requirement for Mad3 ${ }^{\mathrm{SAN}-1}$ as well as BUB-3 indicates that Mad2 ${ }^{\mathrm{MDF}-2}$ is functionally more important. Consistent with this idea, the developmental phenotypes associated with deletion of $\mathrm{Mad}^{\mathrm{SAN}-1}$ are significantly weaker than those resulting from mutations in Mad1 ${ }^{\mathrm{MDF}-1}$ and Mad2 ${ }^{\mathrm{MDF}-2}$, which lead to pronounced defects in germline development and embryo production (Kitagawa and Rose, 1999; Stein et al., 2007). We speculate that in the germline, the core Mad1-Mad2 mechanism may be up-regulated independently of $\mathrm{Mad} 3$ to protect against aneuploidy. It is also possible that, similar to meiosis in budding yeast (Shonn et al., 2003), the Mad1-Mad2 mechanism may provide an additional function important for chromosome segregation. Further work on these two interacting branches of the checkpoint pathway in the context of developmental regulation may provide insight into both the basal checkpoint signaling mechanism and its adaptation in different contexts.

\section{Mad1 $1^{M D F-1} / M a d 2^{M D F-2}$ Versus Mad3 $3^{\text {SAN-1/BUB-3: Two }}$ Branches of the Checkpoint Signaling Pathway}

The most interesting theme emerging from our systematic analysis was the partitioning of the kinetochore-dependent checkpoint signaling pathway into two largely independent branches. Mad2 ${ }^{\mathrm{MDF}-2}$ (and presumably also Mad1 ${ }^{\mathrm{MDF}-1}$ ) accumulate at kinetochores and, in the situation where Mad2 ${ }^{\mathrm{MDF}-2}$ levels are elevated, support kinetochore-dependent checkpoint activation independently of BUB-3 and Mad3 $3^{\mathrm{SAN}-1}$. Conversely, BUB-3 targets to and become enriched at kinetochores in the absence of Mad2 $2^{\mathrm{MDF}-2}$, although in this case no checkpoint signal is generated. The 
independence of Mad1-Mad2 kinetochore localization from Mad3 ${ }^{\text {SAN-1 }}$ is supported by work in yeast (Gillett et al., 2004; Vanoosthuyse et al., 2004) and by BubR1 depletion in human cells (Johnson et al., 2004; Meraldi et al., 2004). Although Mad3 $3^{\text {SAN-1 }}$ does not localize to kinetochores upon checkpoint activation, two lines of evidence support a functional link to BUB-3. First, subtle overexpression of Mad2MDF-2 bypassed depletion of either BUB-3 or Mad3 $3^{\text {SAN-1 }}$. Second, BUB-3 depletion resulted in a significant decrease in GFPMad3 $3^{\mathrm{SAN}-1}$ signal, which suggests that the protein may be destabilized-such an effect is typically observed for proteins that are associated with each other. Together, these results suggest that a core Mad1 ${ }^{\mathrm{MDF}-1}-\mathrm{Mad} 2^{\mathrm{MDF}-2}$ signaling mechanism, which involves conversion of the free "open" form of Mad2 (Mad2-O) to the Cdc20-inhibiting "closed" form (Mad2-C) by a kinetochore-bound Mad1-Mad2 complex (Figure 6B; Musacchio and Salmon, 2007), cooperates with a Mad3 ${ }^{\text {SAN-1 }} /$ BUB-3 dependent cytoplasmic mechanism to inhibit the APC/C (Figure 6B); under normal conditions neither mechanism is sufficient to induce a cell cycle delay. Consistent with this idea, a Bub3-BubR1 complex has been purified from human cells and suggested to inhibit APC/C activity on its own in a manner similar to Mad2 (Tang et al., 2001). The bypass we document here suggests that elevating Mad2 levels enhances Mad2-C formation to a point where the Mad1-Mad2 mechanism is sufficient to induce a kinetochore-dependent cell cycle delay in the absence of the BUB-3/Mad3 ${ }^{\mathrm{SAN}-1}$ branch (Figure $6 \mathrm{~B}$ ). This result suggests that Mad2 levels are limiting for Mad2-C formation in vivo and may be tightly controlled to allow integration of the Mad2-C mechanism with Mad3/BUB-3.

In addition to functioning with $\mathrm{Mad}^{\mathrm{SAN}-1}$ in the cytoplasm, BUB-3 may also act at the kinetochore, because it does enrich there and its depletion reduces the ability of Mad2 ${ }^{\mathrm{MDF}-2}$ to enrich at kinetochores. Because both BUB-1 and Mad3 use a similar and mutually exclusive interaction mechanism to associate with BUB-3 (Wang et al., 2001; Larsen et al., 2007), it is tempting to speculate that there are two pools of BUB-3: a population that enriches at kinetochores complexed with BUB-1 and a population that associates with Mad3 ${ }^{\mathrm{SAN}-1}$ that acts cytoplasmically. It is unclear what effect there is, if any, of kinetochore cycling of BUB-3, presumably via its direct association with BUB-1. It is possible that kinetochore-cycled BUB-3 is modified to potentiate its association with Mad3 $3^{\text {SAN-1 }}$ in the cytoplasm, proving another kinetochore-dependent input.

It is interesting to speculate on why the checkpoint signaling pathway is organized into two interacting branches. One possibility is that synergy between the branches may confer a property to the checkpoint signaling circuit that satisfies its difficult-to-reconcile requirements for potency and lability (Nasmyth, 2005). An attractive alternative possibility is that the Mad1-Mad2 and Mad3/BubR1 mechanisms provide independent inhibitory signals that are responsive to different states-lack of attachment for the Mad1-Mad2 mechanism and lack of tension for the Mad3/ BubR1 mechanism. In support of the latter possibility, a Mad3 phosphorylation site targeted by the error correction kinase Aurora B was recently identified and shown to be specifically required for detecting a defect in tension but not in attachment in budding yeast (King et al., 2007). The two branches may integrate these different inputs to control the stability of Cdc20, which is modulated by checkpoint activation (Pan and Chen, 2004). Further work on the relationship between the Mad1-Mad2 and Mad3/BUB-3 branches may help provide insight into the reasons for this bipartite architecture of the spindle checkpoint pathway.

\section{ACKNOWLEDGMENTS}

We thank members of the Oegema and Desai laboratories for discussions and advice; Paul Maddox for help with imaging; Julien Espeut and Reto Gassmann for help with biochemistry; and Andy Golden, Ann Rose, and Pamela Padilla for strains and other reagents. This work was supported by a University of California, San Diego Genetics training grant (to A. E.), National Institutes of Health grant GM-074215 (to A. D.), and funding from the Ludwig Institute for Cancer Research (to A. D. and K. O.).

\section{REFERENCES}

Abrieu, A., Kahana, J. A., Wood, K. W., and Cleveland, D. W. (2000). CENP-E as an essential component of the mitotic checkpoint in vitro. Cell 102,817-826.

Abrieu, A., Magnaghi-Jaulin, L., Kahana, J. A., Peter, M., Castro, A., Vigneron, S., Lorca, T., Cleveland, D. W., and Labbe, J. C. (2001). Mps1 is a kinetochoreassociated kinase essential for the vertebrate mitotic checkpoint. Cell 106, 83-93.

Bettencourt-Dias, M., and Glover, D. M. (2007). Centrosome biogenesis and function: centrosomics brings new understanding. Nat. Rev. Mol. Cell Biol. 8, 451-463.

Blower, M. D., Daigle, T., Kaufman, T., and Karpen, G. H. (2006). Drosophila CENP-A mutations cause a BubR1-dependent early mitotic delay without normal localization of kinetochore components. PLoS Genet. 2, e110.

Boyarchuk, Y., Salic, A., Dasso, M., and Arnaoutov, A. (2007). Bub1 is essential for assembly of the functional inner centromere. J. Cell Biol. 176, 919-928.

Buchwitz, B. J., Ahmad, K., Moore, L. L., Roth, M. B., and Henikoff, S. (1999) A histone-H3-like protein in C. elegans. Nature 401, 547-548.

Buffin, E., Lefebvre, C., Huang, J., Gagou, M. E., and Karess, R. E. (2005) Recruitment of Mad2 to the kinetochore requires the Rod/Zw10 complex. Curr. Biol. 15, 856-861.

Canman, J. C., Hoffman, D. B., and Salmon, E. D. (2000). The role of pre- and post-anaphase microtubules in the cytokinesis phase of the cell cycle. Curr. Biol. 10, 611-614.

Chan, G. K., Jablonski, S. A., Sudakin, V., Hittle, J. C., and Yen, T. J. (1999) Human BUBR1 is a mitotic checkpoint kinase that monitors CENP-E functions at kinetochores and binds the cyclosome/APC. J. Cell Biol. 146, 941-954.

Cheeseman, I. M., Chappie, J. S., Wilson-Kubalek, E. M., and Desai, A. (2006). The conserved KMN network constitutes the core microtubule-binding site of the kinetochore. Cell 127, 983-997.

Cheeseman, I. M., and Desai, A. (2008). Molecular architecture of the kinetochore-microtubule interface. Nat. Rev. Mol. Cell Biol. 9, 33-46.

Cheeseman, I. M., MacLeod, I., Yates, J. R., 3rd, Oegema, K., and Desai, A. (2005). The CENP-F-like proteins HCP-1 and HCP-2 target CLASP to kinetochores to mediate chromosome segregation. Curr. Biol. 15, 771-777.

Cheeseman, I. M., Niessen, S., Anderson, S., Hyndman, F., Yates, J. R., 3rd, Oegema, K., and Desai, A. (2004). A conserved protein network controls assembly of the outer kinetochore and its ability to sustain tension. Genes Dev. 18, 2255-2268.

Dammermann, A., Maddox, P. S., Desai, A., and Oegema, K. (2008). SAS-4 is recruited to a dynamic structure in newly forming centrioles that is stabilized by the gamma-tubulin-mediated addition of centriolar microtubules. J. Cell Biol. 180, 771-785.

De Antoni, A., et al. (2005). The Mad1/Mad2 complex as a template for Mad2 activation in the spindle assembly checkpoint. Curr. Biol. 15, 214-225.

Desai, A., Rybina, S., Muller-Reichert, T., Shevchenko, A., Hyman, A., and Oegema, K. (2003). KNL-1 directs assembly of the microtubule-binding interface of the kinetochore in C. elegans. Genes Dev. 17, 2421-2435.

Encalada, S. E., Willis, J., Lyczak, R., and Bowerman, B. (2005). A spindle checkpoint functions during mitosis in the early Caenorhabditis elegans embryo. Mol. Biol. Cell 16, 1056-1070.

Fraschini, R., Beretta, A., Sironi, L., Musacchio, A., Lucchini, G., and Piatti, S. (2001). Bub3 interaction with Mad2, Mad3 and Cdc20 is mediated by WD40 repeats and does not require intact kinetochores. EMBO J. 20, 6648-6659.

Gassmann, R., et al. (2008). A new mechanism controlling kinetochore-microtubule interactions revealed by comparison of two dynein-targeting components: SPDL-1 and the Rod/Zwilch/Zw10 complex. Genes Dev. 22, 23852399.

Gillett, E. S., Espelin, C. W., and Sorger, P. K. (2004). Spindle checkpoint proteins and chromosome-microtubule attachment in budding yeast. J. Cell Biol. 164, 535-546. 
Green, R. A., Audhya, A., Pozniakovsky, A., Dammermann, A., Pemble, H., Monen, J., Portier, N., Hyman, A., Desai, A., and Oegema, K. (2008). Expression and imaging of fluorescent proteins in the C. elegans gonad and early embryo. Methods Cell Biol. 85, 179-218.

Hajeri, V. A., Stewart, A. M., Moore, L. L., and Padilla, P. A. (2008). Genetic analysis of the spindle checkpoint genes san-1, mdf-2, bub-3 and the CENP-F homologues hcp-1 and hcp-2 in Caenorhabditis elegans. Cell Div. 3, 6.

Hardwick, K. G., Johnston, R. C., Smith, D. L., and Murray, A. W. (2000). MAD3 encodes a novel component of the spindle checkpoint which interacts with Bub3p, Cdc20p, and Mad2p. J. Cell Biol. 148, 871-882.

Hirose, H., Arasaki, K., Dohmae, N., Takio, K., Hatsuzawa, K., Nagahama, M., Tani, K., Yamamoto, A., Tohyama, M., and Tagaya, M. (2004). Implication of ZW10 in membrane trafficking between the endoplasmic reticulum and Golgi. EMBO J. 23, 1267-1278.

Hoyt, M. A., Totis, L., and Roberts, B. T. (1991). S. cerevisiae genes required for cell cycle arrest in response to loss of microtubule function. Cell 66, 507-517.

Hwang, L. H., Lau, L. F., Smith, D. L., Mistrot, C. A., Hardwick, K. G., Hwang, E. S., Amon, A., and Murray, A. W. (1998). Budding yeast Cdc 20, a target of the spindle checkpoint. Science 279, 1041-1044.

Johnson, V. L., Scott, M. I., Holt, S. V., Hussein, D., and Taylor, S. S. (2004). Bub1 is required for kinetochore localization of BubR1, Cenp-E, Cenp-F and Mad2, and chromosome congression. J. Cell Sci. 117, 1577-1589.

Kapoor, T. M., Mayer, T. U., Coughlin, M. L., and Mitchison, T. J. (2000). Probing spindle assembly mechanisms with monastrol, a small molecule inhibitor of the mitotic kinesin, Eg5. J. Cell Biol. 150, 975-988.

Karess, R. (2005). Rod-Zw10-Zwilch: a key player in the spindle checkpoint. Trends Cell Biol. 15, 386-392.

Kim, S. H., Lin, D. P., Matsumoto, S., Kitazono, A., and Matsumoto, T. (1998) Fission yeast Slp 1, an effector of the Mad2-dependent spindle checkpoint. Science 279, 1045-1047.

King, E. M., Rachidi, N., Morrice, N., Hardwick, K. G., and Stark, M. J. (2007). Ipl1p-dependent phosphorylation of Mad3p is required for the spindle checkpoint response to lack of tension at kinetochores. Genes Dev. 21, 1163-1168.

Kitagawa, R., and Rose, A. M. (1999). Components of the spindle-assembly checkpoint are essential in Caenorhabditis elegans. Nat. Cell Biol. 1, 514-521.

Kitajima, T. S., Hauf, S., Ohsugi, M., Yamamoto, T., and Watanabe, Y. (2005). Human Bub1 defines the persistent cohesion site along the mitotic chromosome by affecting Shugoshin localization. Curr. Biol. 15, 353-359.

Kittler, R., et al. (2007). Genome-scale RNAi profiling of cell division in human tissue culture cells. Nat. Cell Biol. 9, 1401-1412.

Kiyomitsu, T., Obuse, C., and Yanagida, M. (2007). Human Blinkin/AF15q14 is required for chromosome alignment and the mitotic checkpoint through direct interaction with Bub1 and BubR1. Dev. Cell 13, 663-676.

Kops, G. J., Kim, Y., Weaver, B. A., Mao, Y., McLeod, I., Yates, J. R., 3rd, Tagaya, M., and Cleveland, D. W. (2005). ZW10 links mitotic checkpoint signaling to the structural kinetochore. J. Cell Biol. 169, 49-60.

Kurz, T., Pintard, L., Willis, J. H., Hamill, D. R., Gonczy, P., Peter, M., and Bowerman, B. (2002). Cytoskeletal regulation by the Nedd8 ubiquitin-like protein modification pathway. Science 295, 1294-1298.

Larsen, N. A., Al-Bassam, J., Wei, R. R., and Harrison, S. C. (2007). Structural analysis of Bub3 interactions in the mitotic spindle checkpoint. Proc. Natl. Acad. Sci. USA 104, 1201-1206.

Li, R., and Murray, A. W. (1991). Feedback control of mitosis in budding yeast. Cell 66, 519-531.

Luo, X., Tang, Z., Xia, G., Wassmann, K., Matsumoto, T., Rizo, J., and Yu, H. (2004). The Mad2 spindle checkpoint protein has two distinct natively folded states. Nat. Struct. Mol. Biol. 11, 338-345.

Maddox, P. S., Hyndman, F., Monen, J., Oegema, K., and Desai, A. (2007). Functional genomics identifies a Myb domain-containing protein family required for assembly of CENP-A chromatin. J. Cell Biol. 176, 757-763.

Manning, A. L., Ganem, N. J., Bakhoum, S. F., Wagenbach, M., Wordeman, L. and Compton, D. A. (2007). The kinesin-13 proteins Kif2a, Kif2b, and Kif2c/ MCAK have distinct roles during mitosis in human cells. Mol. Biol. Cell 18, 2970-2979.

Mao, Y., Desai, A., and Cleveland, D. W. (2005). Microtubule capture by CENP-E silences BubR1-dependent mitotic checkpoint signaling. J. Cell Biol. $170,873-880$

Mapelli, M., Massimiliano, L., Santaguida, S., and Musacchio, A. (2007). The Mad2 conformational dimer: structure and implications for the spindle assembly checkpoint. Cell 131, 730-743.
McCleland, M. L., Gardner, R. D., Kallio, M. J., Daum, J. R., Gorbsky, G. J., Burke, D. J., and Stukenberg, P. T. (2003). The highly conserved Ndc80 complex is required for kinetochore assembly, chromosome congression, and spindle checkpoint activity. Genes Dev. 17, 101-114.

Meraldi, P., Draviam, V. M., and Sorger, P. K. (2004). Timing and checkpoints in the regulation of mitotic progression. Dev. Cell 7, 45-60.

Millband, D. N., and Hardwick, K. G. (2002). Fission yeast Mad3p is required for Mad2p to inhibit the anaphase-promoting complex and localizes to kinetochores in a Bub1p-, Bub3p-, and Mph1p-dependent manner. Mol. Cell. Biol. $22,2728-2742$.

Minshull, J., Sun, H., Tonks, N. K., and Murray, A. W. (1994). A MAP kinase-dependent spindle assembly checkpoint in Xenopus egg extracts. Cell $79,475-486$

Moore, L. L., and Roth, M. B. (2001). HCP-4, a CENP-C-like protein in Caenorhabditis elegans, is required for resolution of sister centromeres. J. Cell Biol. 153, 1199-1208.

Murray, A. W., and Marks, D. (2001). Can sequencing shed light on cell cycling? Nature 409, 844-846.

Musacchio, A., and Salmon, E. D. (2007). The spindle-assembly checkpoint in space and time. Nat. Rev. Mol. Cell Biol. 8, 379-393.

Nasmyth, K. (2005). How do so few control so many? Cell 120, 739-746.

Nystul, T. G., Goldmark, J. P., Padilla, P. A., and Roth, M. B. (2003). Suspended animation in C. elegans requires the spindle checkpoint. Science 302, $1038-1041$.

O'Connell, K. F., Caron, C., Kopish, K. R., Hurd, D. D., Kemphues, K.J.Li.Y., and White, J. G. (2001). The C. elegans zyg-1 gene encodes a regulator of centrosome duplication with distinct maternal and paternal roles in the embryo. Cell 105, 547-558.

Oegema, K., Desai, A., Rybina, S., Kirkham, M., and Hyman, A. A. (2001) Functional analysis of kinetochore assembly in Caenorhabditis elegans. J. Cell Biol. 153, 1209-1226.

Oegema, K., and Hyman, A. A. (2006). Cell division. WormBook, 1-40.

Pan, J., and Chen R. H. (2004). Spindle checkpoint regulates Cdc20p stability in Saccharomyces cerevisiae. Genes Dev. 18, 1439-1451.

Peters, J. M. (2002). The anaphase-promoting complex: proteolysis in mitosis and beyond. Mol. Cell 9, 931-943.

Praitis, V., Casey, E., Collar, D., and Austin, J. (2001). Creation of low-copy integrated transgenic lines in Caenorhabditis elegans. Genetics 157, 1217-1226. Rajagopalan, H., and Lengauer, C. (2004). Aneuploidy and cancer. Nature 432, 338-341.

Regnier, V., Vagnarelli, P., Fukagawa, T., Zerjal, T., Burns, E., Trouche, D. Earnshaw, W., and Brown, W. (2005). CENP-A is required for accurate chromosome segregation and sustained kinetochore association of BubR1. Mol. Cell. Biol. 25, 3967-3981.

Rieder, C. L., Cole, R. W., Khodjakov, A., and Sluder, G. (1995). The checkpoint delaying anaphase in response to chromosome monoorientation is mediated by an inhibitory signal produced by unattached kinetochores. J. Cell Biol. 130, 941-948.

Rischitor, P. E., May, K. M., and Hardwick, K. G. (2007). Bub1 is a fission yeast kinetochore scaffold protein, and is sufficient to recruit other spindle checkpoint proteins to ectopic sites on chromosomes. PLoS ONE 2, e1342.

Severson, A. F., Hamill, D. R., Carter, J. C., Schumacher, J., and Bowerman, B. (2000). The aurora-related kinase AIR-2 recruits ZEN-4/CeMKLP1 to the mitotic spindle at metaphase and is required for cytokinesis. Curr. Biol. 10, $1162-1171$.

Sharp-Baker, H., and Chen, R. H. (2001). Spindle checkpoint protein Bub1 is required for kinetochore localization of Mad1, Mad2, Bub3, and CENP-E, independently of its kinase activity. J. Cell Biol. 153, 1239-1250.

Shonn, M. A., Murray, A. L., and Murray, A. W. (2003). Spindle checkpoint component Mad2 contributes to bioorientation of homologous chromosomes. Curr. Biol. 13, 1979-1984

Sironi, L., Mapelli, M., Knapp, S., De Antoni, A., Jeang, K. T., and Musacchio, A. (2002). Crystal structure of the tetrameric Mad1-Mad2 core complex: implications of a 'safety belt' binding mechanism for the spindle checkpoint. EMBO J. 21, 2496-2506

Stein, K. K., Davis, E. S., Hays, T., and Golden, A. (2007). Components of the spindle assembly checkpoint regulate the anaphase-promoting complex during meiosis in Caenorhabditis elegans. Genetics 175, 107-123.

Sudakin, V., Chan, G. K., and Yen, T. J. (2001). Checkpoint inhibition of the APC/C in HeLa cells is mediated by a complex of BUBR1, BUB3, CDC20, and MAD2. J. Cell Biol. 154, 925-936. 
Tang, Z., Bharadwaj, R., Li, B., and Yu, H. (2001). Mad2-Independent inhibition of APCCdc20 by the mitotic checkpoint protein BubR1. Dev. Cell 1, 227-237.

Tang, Z., Shu, H., Oncel, D., Chen, S., and Yu, H. (2004a). Phosphorylation of Cdc20 by Bub1 provides a catalytic mechanism for APC/C inhibition by the spindle checkpoint. Mol. Cell 16, 387-397.

Tang, Z., Sun, Y., Harley, S. E., Zou, H., and Yu, H. (2004b). Human Bub1 protects centromeric sister-chromatid cohesion through Shugoshin during mitosis. Proc. Natl. Acad. Sci. USA 101, 18012-18017.

Tarailo, M., Tarailo, S., and Rose, A. M. (2007). Synthetic lethal interactions identify phenotypic "interologs" of the spindle assembly checkpoint components. Genetics 177, 2525-2530.

Vanoosthuyse, V., Valsdottir, R., Javerzat, J. P., and Hardwick, K. G. (2004) Kinetochore targeting of fission yeast Mad and Bub proteins is essential for spindle checkpoint function but not for all chromosome segregation roles of Bub1p. Mol. Cell. Biol. 24, 9786-9801.

Vaur, S., Cubizolles, F., Plane, G., Genier, S., Rabitsch, P. K., Gregan, J., Nasmyth, K., Vanoosthuyse, V., Hardwick, K. G., and Javerzat, J. P. (2005). Control of Shugoshin function during fission-yeast meiosis. Curr. Biol. 15, $2263-2270$

Vink, M., Simonetta, M., Transidico, P., Ferrari, K., Mapelli, M., De Antoni, A., Massimiliano, L., Ciliberto, A., Faretta, M., Salmon, E. D., and Musacchio, A. (2006). In vitro FRAP identifies the minimal requirements for Mad2 kinetochore dynamics. Curr. Biol. 16, 755-766.
Wang, X., Babu, J. R., Harden, J. M., Jablonski, S. A., Gazi, M. H., Lingle, W. L., de Groen, P. C., Yen, T. J., and van Deursen, J. M. (2001). The mitotic checkpoint protein hBUB3 and the mRNA export factor hRAE1 interact with GLE2p-binding sequence (GLEBS)-containing proteins. J. Biol. Chem. 276, 26559-26567.

Warren, C. D., Brady, D. M., Johnston, R. C., Hanna, J. S., Hardwick, K. G., and Spencer, F. A. (2002). Distinct chromosome segregation roles for spindle checkpoint proteins. Mol. Biol. Cell 13, 3029-3041.

Watanabe, S., Yamamoto, T. G., and Kitagawa, R. (2008). Spindle assembly checkpoint gene mdf-1 regulates germ cell proliferation in response to nutrition signals in C. elegans. EMBO J. 27, 1085-1096.

Weiss, E., and Winey, M. (1996). The Saccharomyces cerevisiae spindle pole body duplication gene MPS1 is part of a mitotic checkpoint. J. Cell Biol. 132, 111-123.

Williams, B. C., Li, Z., Liu, S., Williams, E. V., Leung, G., Yen, T. J., and Goldberg, M. L. (2003). Zwilch, a new component of the ZW10/ROD complex required for kinetochore functions. Mol. Biol. Cell 14, 1379-1391.

Yang, M., Li, B., Liu, C. J., Tomchick, D. R., Machius, M., Rizo, J., Yu, H., and Luo, X. (2008). Insights into mad2 regulation in the spindle checkpoint revealed by the crystal structure of the symmetric mad2 dimer. PLoS Biol. 6, e50.

$\mathrm{Yu}, \mathrm{H}$. (2002). Regulation of APC-Cdc20 by the spindle checkpoint. Curr. Opin. Cell Biol. 14, 706-714.

Yu, H. (2007). Cdc 20, a WD40 activator for a cell cycle degradation machine Mol. Cell 27, 3-16. 